\title{
Echoes of Emotions Past: How Neuromodulators Determine What We Recollect
}

\author{
David Clewett, ${ }^{{ }^{\wedge}}$ and ${ }^{-}$Vishnu P. Murty ${ }^{\wedge}$
}

https://doi.org/10.1523/ENEURO.0108-18.2019

${ }^{1}$ Department of Psychology, New York University, New York City, New York, 10003 and ${ }^{2}$ Department of Psychology, Temple University, Philadelphia, PA, 19122

\begin{abstract}
We tend to re-live emotional experiences more richly in memory than more mundane experiences. According to one recent neurocognitive model of emotional memory, negative events may be encoded with a larger amount of sensory information than neutral and positive events. As a result, there may be more perceptual information available to reconstruct these events at retrieval, leading to memory reinstatement patterns that correspond with greater memory vividness and sense of recollection for negative events. In this commentary, we offer an alternative perspective on how emotion may influence such sensory cortex reinstatement that focuses on engagement of the noradrenergic (NE) and dopaminergic (DA) systems rather than valence. Specifically, we propose that arousal-related locus coeruleus-norepinephrine (LC-NE) system activation promotes the prioritization of the most salient features of an emotional experience in memory. Thus, a select few details may drive lower-level sensory cortical activity and a stronger sense of recollection for arousing events. By contrast, states of high behavioral activation, including novelty-seeking and exploration, may recruit the DA system to broaden the scope of cognitive processing and integrate multiple event aspects in memory. These more integrated memory representations may be reflected in higher-order cortical reinstatement at retrieval. Thus, the balance between activation in these neuromodulatory systems at encoding, rather than the valence of the event, may ultimately determine the quality of emotional memory recollection and neural reinstatement.
\end{abstract}

Key words: context; dopamine; emotion; episodic memory; norepinephrine; recollection

\section{Significance Statement}

We tend to re-live emotional events more richly in memory than mundane experiences. Recent research suggests that emotional memory enhancements, particularly for negative events, emerge from greater reactivation of sensory information during retrieval. Here, we offer an account of how emotion influences such sensory cortex reinstatement that focuses on how activation of the noradrenergic (NE) and dopaminergic (DA) systems may impact the quality of recollection. We propose that NE system activation promotes the prioritization of the most salient features of an event in memory, whereas DA system activation promotes the integration of multiple event features in memory. Thus, the balance between activation in these systems might determine how we encode and later re-experience our most significant events.

Emotional events are resistant to forgetting and are vividly remembered (LaBar and Cabeza, 2006; Markovic et al., 2014; Yonelinas and Ritchey, 2015). This privileged status of emotional experiences in memory is highly adap-

Received April 13, 2018; accepted February 14, 2019; First published March 4, 2019.

The authors declare no competing financial interests. tive, because it promotes future behaviors that help us avoid threat and maximize well-being (Shohamy and Adcock, 2010; Eaton and Anderson, 2018). Years of psychology and neuroscience research have uncovered adaptive brain mechanisms that preferentially support the 
formation and storage of emotional memories. However, these neurocognitive models have focused disproportionately on earlier stages of memory processing, including encoding and consolidation (for review, see McGaugh, 2004; LaBar and Cabeza, 2006), with less attention being given to emotion's downstream effects on later memory retrieval.

In their recent Negative Emotional Valence Enhances Recapitulation (NEVER) model, Bowen et al. (2018) theorize a significant role for retrieval-related processes in facilitating emotional memory (Bowen et al., 2018). Specifically, they propose that negative memories contain more sensory information from the original event than both neutral and positive memories. These perceptuallyrich mental representations are then reinstated in the brain during retrieval, resulting in more detailed and vivid memories for negative events. The authors also suggest that negative valence strengthens the links between different stages of episodic memory, such that more sensory-focused encoding leads to preferential reactivation of these perceptual details during offline consolidation and, consequently, later recollection. The NEVER model thereby differentiates itself from prior models of emotional memory by: (1) highlighting a prominent role for retrieval-related sensory processing in facilitating emotional memory, and (2) emphasizing that these mnemonic advantages are specific to negative events.

Although this framework offers a compelling account of the phenomenology of emotional memory recollection, it is somewhat incomplete. Here, we propose some important considerations for the NEVER model and discuss some methodological caveats that warrant further attention. Building on this critique, we offer another perspective of how emotion influences retrieval-related sensory cortex reinstatement, or "recapitulation," that focuses on how activity in the noradrenergic (NE) and, possibly, dopaminergic (DA) systems may impact emotional memory formation and the quality of recollection. Our neuromodulation-based model expands upon NEVER by clarifying which aspects of emotional experiences are being recapitulated during retrieval. It also specifies multiple neural mechanisms by which neural reinstatement may occur, including several factors, such as physiological arousal and behavioral activation, that determine these differential patterns of engagement. By filling in these knowledge gaps, we aim to provide a more holistic view of how emotion influences what we remember about our most significant experiences.

This project was funded by a National Alliance for Research on Schizophrenia and Depression Young Investigator Award and $\mathrm{K} 01 \mathrm{MH} 111991$ (to V.P.M.), and F32 MH114536 (to D.C.).

${ }^{\wedge}$ D.C. and V.P.M. contributed equally to this work.

Correspondence should be addressed to Vishnu P. Murty at vishnu. murty@temple.edu.

https://doi.org/10.1523/ENEURO.0108-18.2019

Copyright (C) 2019 Clewett and Murty

This is an open-access article distributed under the terms of the Creative Commons Attribution 4.0 International license, which permits unrestricted use, distribution and reproduction in any medium provided that the original work is properly attributed.

\section{Brain mechanisms that support the recollection of past events}

When memories are brought to mind, it can often feel as if those events are being re-experienced in the moment (Tulving and Markowitsch, 1998). Seminal neurocognitive models of recollection propose that the extent to which we re-live prior events may be driven by greater correspondence between retrieval-related brain activity and the neural processes that were engaged at encoding (McClelland et al., 1995; Danker and Anderson, 2010). At the neural level, this idea is supported by neuroimaging research showing that successfully retrieving prior episodes is associated with reactivation of neural memory and sensory traces first laid down at encoding (Nyberg et al., 2000; Wheeler et al., 2000; Danker and Anderson, 2010; Mack and Preston, 2016; Tompary et al., 2016; Danker et al., 2017).

Emotional memories tend to be highly vivid, suggesting that they may contain richer sensory information that is available for reconstruction, or recollection, during later retrieval. Behavioral evidence suggests that negative emotional events, in particular, are remembered with greater perceptual detail than neutral and sometimes positive events (Kensinger, 2007). Supporting this notion, human fMRI studies show that encoding negative experiences relates to greater activity in perceptual processing regions, including visual cortex, suggesting that negative memories may contain more perceptual details than other memories (Kensinger et al., 2007a,b; Mickley and Kensinger, 2008).

Until recently, the majority of research has focused on how emotional memories garner greater attention than neutral experiences and are preferentially processed during encoding. This has left an important knowledge gap concerning how emotion impacts memory retrieval processes downstream, which has important implications for the wellbeing of both healthy individuals and individuals with emotion or anxiety disorders when they re-live their most aversive event. In this section, we outline key predictions of the NEVER model, which was proposed to fill this critical gap.

\section{The NEVER model: core tenets and primary empirical evidence}

According to the NEVER model, emotion-related biases in perceptual processing may re-emerge during retrieval, leading to a more vivid sense of recollection. Building on earlier investigations targeting neural recapitulation effects with neutral stimuli, Bowen et al. (2018) hypothesized that there is greater encoding-retrieval overlap in brain activity within early sensory processing regions, particularly for negative stimuli.

In an initial test of this idea, Kark and Kensinger (2015) used fMRI to examine brain activity patterns associated with encoding and retrieving emotional and neutral experiences. During encoding, participants viewed colored photographs of negative, positive, and neutral scenes along with corresponding black-and-white line drawing of those pictures. During retrieval, participants had to decide whether line drawings were previously associated with a 
colored image or whether they had never been seen before. One advantage of this retrieval manipulation is that it avoids reintroducing strong arousal/emotional states by using the emotional associate as the memory cue. Thus, any patterns of reinstatement can be more clearly interpreted as mental reactivation as opposed to purely sensory properties of the cue.

Although there were no emotional memory enhancements in recognition memory, conjunction analyses performed on the $\mathrm{fMRI}$ data revealed overlapping patterns of activation between encoding and retrieval. These analyses revealed three key findings: (1) in large portions of lateral occipito-temporal cortex, there was large spatial overlap across encoding and retrieval for successful memory-related brain activity for both positive and negative images (Fig. 1A); (2) in inferior temporal gyrus, there was significantly greater spatial overlap between the encoding and retrieval of negative versus positive images; and (3) for negative items, there was greater spatial overlap between encoding and retrieval in fusiform gyrus, a region that was also more functionally coupled with amygdala activity during successful encoding. These findings suggest that negative emotional memories may be recapitulated to a larger extent than positive memories in regions within ventral occipito-temporal cortex, and this sensory recapitulation of negative stimuli may be mediated by amygdala modulation at encoding.

In a more recent study, Bowen and Kensinger (2017) designed an $\mathrm{fMRI}$ paradigm that could more directly test this relationship between emotional memory recollection and neural recapitulation. During encoding, participants viewed neutral words that were paired with negative, positive, and neutral images. These images consisted of either faces or scenes. During retrieval, participants were shown the neutral word cue and were asked to retrieve the accompanying picture. Like the prior study, the strength of this approach is that reactivation differences between valences cannot be attributed to sensory activation driven by the emotional images themselves. Rather, the word cues are equally devoid of strong visual input. Behaviorally, memory was enhanced for neutral words paired with negative scenes compared to positive or neutral scenes; however, there was no valence differences for words paired with emotional or neutral faces. Conjunction fMRI analyses revealed qualitatively greater recollection-related encoding-retrieval spatial overlap in visual occipito-temporal cortical activity and amygdala for negative contexts than positive and neutral contexts (Fig. 1B), although this pattern was not seen in category-selective visual cortex. This pattern of findings remained when the authors controlled for stimulus arousal across valence (comparing positive scenes vs negative faces) and lowerlevel stimulus features (comparing positive vs negative faces).

Together, the findings from these fMRI studies lend initial support to the idea that when negative emotional memories are successfully remembered (as, on average, there were no behavioral effects of emotion on memory), they may be recollected with greater perceptual information than positive and neutral memories. Inspired by these findings, the NEVER model was put forth to address the underappreciated role of emotional valence on episodic memory. The primary aims of this brain-based model are to: (1) shift the focus of emotional memory mechanisms beyond regions traditionally implicated in emotional memory, including the amygdala and hippocampus (Dolcos et al., 2004; Ritchey et al., 2008; Murty et al., 2010), to capture the local reinstatement of sensory-rich negative memory representations at retrieval; (2) encourage a broader appreciation of non-arousal-related factors that may enhance memory vividness and recollection; and (3) place a larger emphasis on how different stages of episodic memory processing interact, with a particular focus on how encoding biases relate to subsequent patterns of post-encoding memory reactivation and retrieval.

\section{A new perspective of emotional memory and neural reinstatement}

The NEVER model offers an interesting account of the neural factors that facilitate emotional memory and its persistence. But arguably, the bulk of the literature still implicates physiological arousal as a driving force behind the modulation of recollection effects. Indeed, the critical role of physiological arousal in modulating episodic memory remains a central feature of many neurocognitive theories of emotional memory (see Table 1 in Bowen et al., 2018). While it is possible that valence effects are still at play, there are notable caveats in NEVER's empirical studies that need to be addressed, including deconfounding the effects of valence and arousal on neural recapitulation. Importantly, the effects of positive valence on recollection are quite variable (see Table 2 in Bowen et al., 2018). For instance, in some cases, behavioral work shows that positive events are also encoded with great perceptual detail, perhaps even more than negative stimuli (Talarico et al., 2009).

A challenge to understanding the role of valence in emotional memory, has been operationalizing the influence of arousal on the formation of positive memories. Despite efforts to experimentally control for arousal differences between positive and negative emotional stimuli, participants' ratings sometimes deviate from normative arousal ratings [e.g., international affective picture system (IAPS) images; Lang and Bradley, 2007]. Due to this, it may be the case that arousal-related confounds cloud the interpretation of valence differences that have been reported across many studies of emotional memory. In prior research, these arousal-related confounds have emerged in several ways. First, in some cases, arousal was not experimentally controlled between positive and negative emotional stimuli (Maratos et al., 2001). In other cases, differences in subjects' self-reported arousal persisted even though arousal levels were matched between positive and negative emotional stimuli beforehand (Kark and Kensinger, 2015). Because negative stimuli tend to be much more arousing than positive stimuli, one possibility is that valence differences emerge due to arousal levels being relatively moderate. In other words, to be able to equate arousal levels across valences, the arousal level of selected negative stimuli may be relatively low and there- 
A

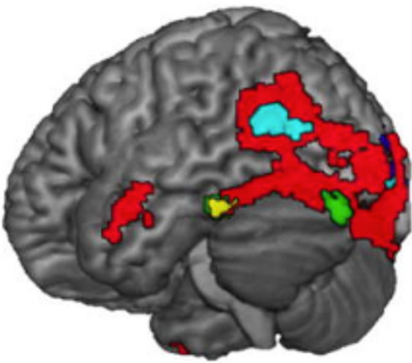

Noft hemisphere

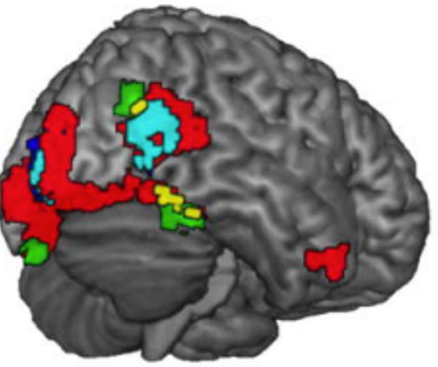

right hemisphere
Encoding $\cap$ Retrieval:

$\square$ Negative Hits > Neutral Hits

Positive Hits > Neutral Hits

$\square$ Spatial Overlap

Relation to emotional memory

enhancement:

$\square$ Emotional Hits at retrieval

$\square$ Spatial overlap with red box

B

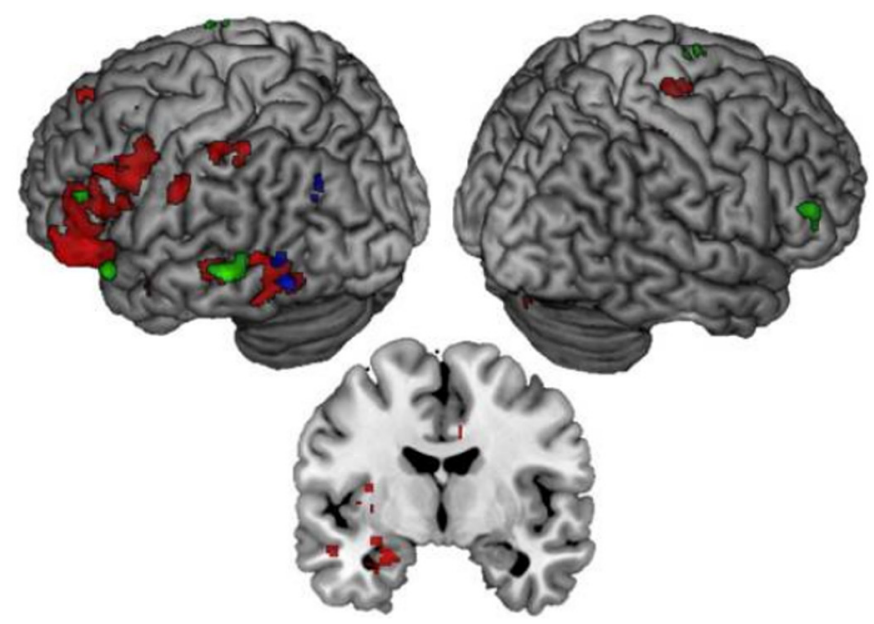

C

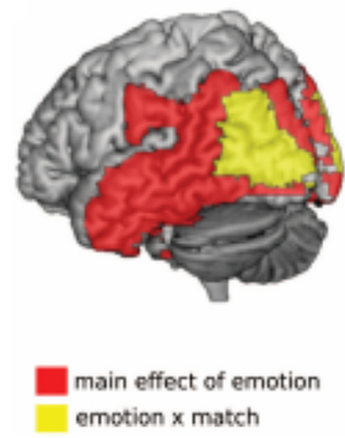

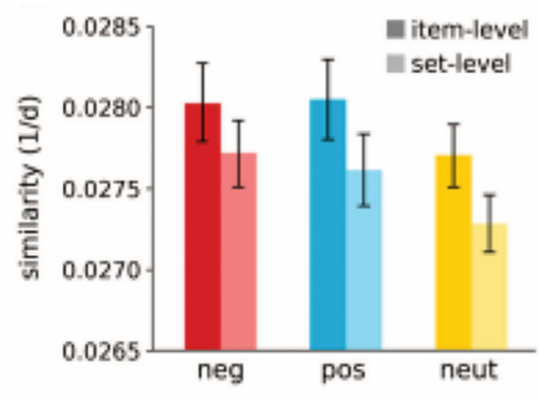

Middle Occipital Gyrus

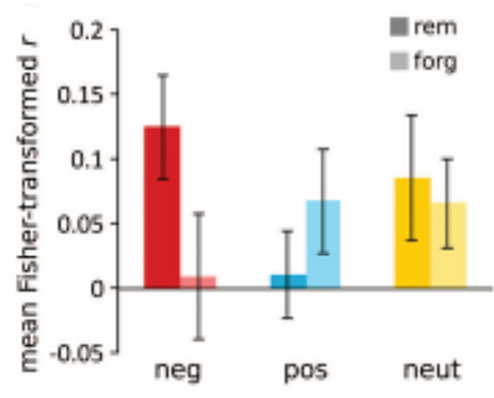

MOG - right Amygdala

Figure 1. Empirical evidence of emotion-enhanced sensory cortex and amygdala recapitulation during memory retrieval. $\boldsymbol{A}$, Conjunction fMRI analyses of brain activity associated with successful memory during encoding and retrieval revealed significant spatial overlap (i.e., recapitulation) in ventral temporo-occipital cortex for negative and positive emotional images (red, blue and turquoise clusters). The spatial extent of recapitulation across the visual stream was greater for negative (red clusters) than positive emotional stimuli (blue clusters). Areas associated with emotion-related recapitulation were also correlated with emotional enhancements (not differentiated by valence) in behavior (data and figure from Kark and Kensinger, 2015). B, Extending these findings, one recent $\mathrm{fMRI}$ study using encoding-retrieval conjunction analyses revealed greater extent of recollection-specific activity (i.e., "remember hits" vs "know hits") across PFC, temporal cortex, and occipital cortex for negative images (red clusters). Similar neural recapitulation patterns were observed for positive (blue clusters) and neutral stimuli (green clusters) in ventral occipito-temporal cortex, but to a quantitatively lesser extent than negative stimuli. Recollection of negative valence stimuli was also associated with enhanced recapitulation activity in the amygdala. Because neutral word cues were used to cue "remember" or "know" memory decisions, these neural patterns are interpreted as evidence of the recapitulation of the perceptual content of emotional memories (data and figure from Bowen and Kensinger, 2017). C, In a separate fMRI study, Ritchey et al. (2013) used pattern similarity analyses to examine correspondence between item- versus set-level activation patterns during encoding and retrieval of positive, negative, and neutral stimuli. The results revealed similar patterns of memory-related recapitulation in middle occipital gyrus for positive (blue bars) and negative (red bars) versus neutral stimuli (yellow bars) for item-specific representations. Further, the degree of encoding-retrieval similarity in middle occipital gyrus was positively correlated with retrieval-related activity in the amygdala, but only for negative valence stimuli (data and figure from Ritchey et al., 2013). 
fore fail to capture a robust effect of high-arousal states on declarative memory. Finally, in one fMRI study, stimuli drawn from different visual categories (i.e., negative faces, and positive scenes) had to be compared to control for arousal (Bowen and Kensinger, 2017).

Based on issues like these, we believe it is premature to rule out the possibility that arousal mechanisms, rather than valence, mediate emotion-related effects on reactivation in early sensory cortex. This idea is further reinforced by a prior fMRI study reporting equivalent levels of sensory recapitulation during retrieval for both positive and negative memoranda in general. But valence differences emerged when examining amygdala modulation, which may reflect variability in arousal across emotional valences (Ritchey et al., 2013; Fig. 1C).

To address these issues, we propose a new model that considers emotional memory reinstatement processes within the broader context of the emotion, episodic memory, and motivation literatures. Our goal is to move beyond a valence-based interpretation of emotional memory recollection, per se, to encompass other cognitive and emotional factors that impact various stages of episodic memory. The main points of our framework can be summarized as follows:

(1) Sympathetic arousal, rather than negative valence, drives selective memory of prioritized and item-intrinsic features during encoding, leading to enhanced subjective sense of recollection and memory vividness. This cognitive narrowing effect also results in greater reinstatement patterns in relatively lower-level sensory cortical regions during retrieval. Further, this process may be driven by arousal-related NE system activity and amygdala activity at encoding and consolidation, as well as by amygdala (re)activation at retrieval.

(2) Behavioral activation, defined as increased behavioral vigor or novelty-seeking, may engage alternative mechanisms that reflect the formation of integrative, flexible memory representations. This memory process may be signified by reinstatement effects in relatively higherorder cortex, such as anterior portions of the ventral visual stream as well as lateral and medial prefrontal cortex (PFC). One possibility is that mesolimbic DA system activation supports these more integrative forms of memory and high-order reactivation.

(3) Focusing on arousal and behavioral activation rather than valence may provide a better schema for understanding enhanced neural reinstatement patterns for emotional memories. Critically, emotional valence may be inter-related with arousal and behavioral activation, such that negative stimuli are biased towards eliciting greater arousal and positive stimuli are biased towards eliciting behavioral activation. However, in many behavioral contexts, negative stimuli might evoke behavioral activation, and positive stimuli may elicit high degrees of arousal. Thus, our goal is to move beyond valence effects to consider alternative factors that shape patterns of neural reactivation/reinstatement and its manifestation of memory.

\section{What types of sensory information are reinstated during emotional memory retrieval?}

The NEVER model proposes that sensory features of a negative emotional event are reactivated in sensory cortex during retrieval, with the implication that this recapitulation brings to mind the details and vividness of the original event. We broaden this interpretation by proposing that these recapitulation effects reflect certain aspects of the original event in which only the most salient item and episodic details are stored in memory and later reinstated. Current data suggest that this process may be predominantly driven by increased emotional arousal.

A large body of research shows that recollection, defined as remembering an item with accompanying episodic information, is better for emotional experiences than for neutral experiences. However, it has long been known that the impact of emotion on cognition is highly selective and does not benefit all aspects of episodic memory (Anderson et al., 2006; Rimmele et al., 2011; Bisby and Burgess, 2013; Steinmetz and Kensinger, 2013; Madan et al., 2017). This issue raises the critical question of which episodic details of emotional experiences are being reinstated and recollected at retrieval.

Studies that query source memory (e.g., an item's timing, location, or perceptual features) may provide some clues as to the episodic content of emotional memories. A wealth of evidence suggests that memory is selectively enhanced for emotional items and their intrinsic details (Yonelinas and Ritchey, 2015), such as their location or color, whereas memory for spatially or temporally adjacent contextual information is impaired (Christianson and Loftus, 1991; Kensinger, 2007; Mather, 2007; Touryan et al., 2007; Mather and Sutherland, 2011; Steinmetz and Kensinger, 2013; Bisby and Burgess, 2017). Further, prior work has linked such emotional memory selectivity to increased arousal levels rather than valence (Mather and Nesmith, 2008), consistent with the idea that arousal focuses limited mental resources towards processing a select few details of an emotional event (Easterbrook, 1959).

One possibility is the strength of these specific memory traces modulates subjective aspects of emotional memories. Emotion enhances the subjective sense of remembering, such that participants tend to report higher subjective confidence and vividness for high arousal, emotional memories (Ochsner, 2000; Kensinger and Corkin, 2003; Sharot et al., 2004; Touryan et al., 2007; Ritchey et al., 2008; Todd et al., 2013b). Yet this increased confidence does not always correspond with greater memory accuracy, at least for all aspects of an emotional experience. Rather, subjective memory confidence appears to relate specifically to successful retrieval of emotional item-intrinsic details, such as the location and timing of an emotional stimulus (Rimmele et al., 2012) and not more peripheral sensory information, such as the surrounding context (Rimmele et al., 2011).

Considering that highly confident memory decisions are closely tied to memory for emotional item-intrinsic information, it may be the case that enhanced recollection for emotional events is not driven by the amount of sensory 
details that are recovered. Instead, vividly re-experiencing a prior emotional event could be driven by especially strong memory representations of select details (for a similar proposal, see Kensinger, 2009). Indeed, higher subjective memory response encountered after an arousing negative event have been linked to enhanced memory for intrinsic sensory features (e.g., color) of those neutral items (Xie and Zhang, 2017). Eye-tracking evidence also shows that recollection judgements for high-arousal negative images are predicted by more clustered rather than distributed fixations during encoding, suggesting that specific features of these events drive subsequent recollection (Sharot et al., 2008). Together, these data are consistent with evidence that emotional arousal amplifies the effects of priority (e.g., goal-relevance, bottom-up salience) in perception and memory, leading to the selective encoding of highly salient information (Mather and Sutherland, 2011; Sakaki et al., 2014).

In sum, we argue that for high arousal memoranda, which are often tested in the domain of negative valence, the subjective sense of remembering is driven by a narrowing of attention towards only the most central, emotionally-laden details of an experience. We note that this more focused cognitive processing induced by arousal should incorporate neutral information insofar as those neutral associates are task-relevant and actively prioritized under emotional circumstances. In these situations, neutral cues will be bound more strongly to their associated emotional items in memory, and can act as an effective trigger to recall (and/or recapitulate) emotional events (for review, see Mather and Sutherland, 2011; Murray and Kensinger, 2013; Sakaki et al., 2014). For instance, both positive and negative background scenes can enhance memory for foreground neutral objects when participants are instructed to unitize those inputs (e.g., imagine them interacting) during encoding (Ventura-Bort et al., 2016).

To interpret the true nature of emotion-related sensory recapitulation, then, it is critical to consider where attention is allocated during encoding, and, second, whether participants' memory is assessed for the salient or nonsalient features of that emotional event. This feature of emotional memory selectivity suggests that sensory recapitulation reflects a biased representation of the most salient event features, perhaps at the expense of the surrounding or irrelevant perceptual information.

\section{Is the preferential processing and retrieval of emotional item-intrinsic information dependent on the amygdala?}

Next, we consider how emotional arousal may contribute to greater episodic memory selectivity during emotional memory. Much research implicates amygdala processes in modulating the quality of emotional memory recollection, particularly by promoting the encoding of specific details of arousing events (Sharot et al., 2004; Phelps and Sharot, 2008). Greater amygdala activity at encoding has been specifically linked to successful item recognition and higher subjective memory vividness for positive and negative images, but not to the successful memory of peripheral contextual information (Dougal et al., 2007; Kensinger et al., 2007a,b). Lesion data corroborate this role of the amygdala in selective memory processes. Patients with amygdala damage show selective memory deficits for the central but not peripheral details of an emotional event (Adolphs et al., 2001, 2005; Yonelinas and Ritchey, 2015). Thus, amygdala activity at encoding may lead to more vivid recollections by forming especially strong memories of highly specific aspects of an emotional event.

At the network level, this memory selectivity may result from the amygdala modulating early sensory cortical activity (Todd et al., 2012, 2013b). For instance, for high-arousal negative stimuli, greater encoding-related amygdala functional connectivity with the fusiform gyrus, a region associated with processing item-exemplar information (GaroffEaton et al., 2006), was also associated with greater sensory recapitulation (encoding-retrieval overlap) in fusiform gyrus (Kark and Kensinger, 2015). This suggests a link between early amygdala tuning processes in sensory cortex during emotional memory formation and local sensory cortex reinstatement effects.

The notion that negative valence may modulate these amygdala effects is partially motivated by $\mathrm{fMRI}$ evidence using effective (e.g., directional) connectivity analyses (Mickley Steinmetz et al., 2010). This study showed that patterns of amygdala modulation at encoding diverge at different levels of arousal in a valence-dependent manner. Specifically, amygdala modulation of middle occipital gyrus activity was increased for high-arousal negative stimuli but decreased for high-arousal positive stimuli. But it is important to note that the opposite valencedependent flip was observed in the amygdala's modulation of fusiform gyrus activity: whereas the amygdala's influence over activity in this higher-order sensory region was increased for high-arousal positive images, it was decreased for high-arousal negative images. This again raises the question of what types of sensory information are being recapitulated by amygdala engagement. Without querying which specific episodic details are being remembered and linking this to brain activation patterns, it is difficult to interpret what non-category-selective patterns of cortical activation mean for models of sensory recapitulation.

Emotion-related encoding biases may also propagate downstream and manifest in patterns of post-encoding functional connectivity, particularly when arousal levels remain elevated. The way emotional information is encoded therefore determines how emotional experiences are subsequently consolidated and retrieved. The amygdala also appears to shape emotional memory in this regard, either exhibiting increased local activity or more distributed patterns of modulation during the encoding, consolidation, and retrieval of emotional memories (Buchanan, 2007). Fear learning paradigms, for example, offer a robust way of manipulating arousal responses that facilitate memory. Across several fMRI studies, increases in amygdala functional connectivity with the hippocampus (de Voogd et al., 2016; Hermans et al., 2017) and categoryselective cortex (Hermans et al., 2017) have been shown to 
persist into post-conditioning periods of wakeful rest. These connectivity patterns also predicted spontaneous fear recovery $24 \mathrm{~h}$ later. The findings lend support to influential theories positing that the amygdala modulates systemslevel consolidation processes that enhances the long-term retention of emotionally arousing memories (McGaugh and Roozendaal, 2002; McGaugh, 2000).

Research also suggests that amygdala activity contributes to emotional memory recollection, in part through reinstatement (Bowen and Kensinger, 2017; Fig. 1B). One possibility, however, is that this recapitulation relates more closely to high arousal rather than negative valence. For instance, amygdala activity during memory retrieval tracks individual self-reports of arousal on a stimulus-by-stimulus basis (Dew et al., 2014). This suggests arousal-related activation of the amygdala promotes more sensory-focused attention and encoding processes in a valence-invariant fashion. The specific conditions that give rise to valence differences at retrieval require additional research, given that amygdala activity at retrieval has also been reported for positive stimuli (Buchanan, 2007).

Amygdala processes might also modulate the subjective quality of emotional memory recollection during the retrieval act itself, but not necessarily memory accuracy (Sharot et al., 2004). In fact, amygdala activity may give rise to a false sense of remembering, especially when participants must retrieve or recollect contextual information that is peripheral to the to-be-encoded item, such as a neutral or arousing negative background (Takashima et al., 2016). Across multiple fMRI studies, amygdala and sensory cortex activity in response to contexts or items deemed arousing, including negative emotional items, are either unrelated to or negatively associated with subsequent source memory (Smith et al., 2006; Takashima et al., 2016). These findings are consistent with behavioral work showing that source memory is impaired for information that is peripheral to an emotional stimulus, despite individuals endorsing an emotional stimulus as being remembered (Rimmele et al., 2011). Thus, some aspects of retrieval-related sensory cortex and amygdala activity may represent noisy or inaccurate cognitive/sensory representations, especially for peripheral details that may have been poorly encoded to begin with.

In summary, current findings point to a role of arousaldriven amygdala activation and modulation in encoding, consolidating, and retrieving emotionally arousing memories. However, neural reinstatement effects may be more nuanced in that they support the accurate recollection of prioritized sensory features but not less salient peripheral features. These results also underscore the importance of querying recollection for both central and peripheral details of an emotional event, and linking different aspect of memory performance to brain activity patterns across different stages of episodic memory.

\section{Does neuromodulation explain differences in the prioritization of sensory features in emotional memory?}

To better understand how emotion influences neural reinstatement processes in the brain, we propose that it is important to consider the valence-invariant role of neuromodulatory arousal systems in supporting emotional memory encoding. It is well established that arousalinduced activation of the locus coeruleus-norepinephrine (LC-NE) system contributes to the superior processing, encoding, and consolidation of emotional experiences in long-term episodic memory (Roozendaal et al., 1996, 2008; Southwick et al., 2002; McGaugh, 2004; Strange and Dolan, 2004; Segal and Cahill, 2009). Through its diffuse neuronal projections to most of the brain, the LC is well positioned to modulate sensory cortical and amygdala processes during highly arousing and salient events (Berridge and Waterhouse, 2003; Sara, 2009). Such modulation under arousal may be particularly important in shaping the contents of memory during encoding. Human memory studies utilizing pharmacological and genetic approaches have shown that putative increases in NE tone at encoding but not retrieval enhance the subjective recollection and vividness of emotional memory later on (Rimmele et al., 2016; Fig. 2).

Research suggests that amygdala-centric mechanism of emotional memory may be in large part driven by the release of NE both during emotional encoding and consolidation (McGaugh, 2004, 2015; Strange and Dolan, 2004). But these selective arousal-related memory enhancements may come at the expense of processing spatially or temporally adjacent neutral information, suggesting that NE modulation of the amygdala biases attention and memory processes to favor emotional over neutral information (Strange and Dolan, 2004; Markovic et al., 2014). These empirical findings lend strong support to influential neurobiological models of emotional memory, which posit that NE signaling under arousal biases the amygdala to preferentially enhance encoding and long-term storage of emotionally salient mental representations in the sensory cortex (e.g., visual cortex), the medial temporal lobe (e.g., entorhinal cortex), and hippocampus (McGaugh, 2004; Roozendaal et al., 2008; Tully and Bolshakov, 2010; Mclntyre et al., 2012; Markovic et al., 2014; Mather et al., 2016). Under emotional arousal, elevated levels of NE also enhance the vividness of perception, in part through increased amygdala influence over sensory cortex activity (Todd et al., 2013b, 2015).

Activation of the LC-NE system has also been observed during emotional memory retrieval. In one fMRI study, greater functional connectivity between the amygdala and LC was associated with successful retrieval of neutral items encoded in aversive contexts (Sterpenich et al., 2006). Further, activity in the LC activity during successful memory retrieval varied according to the level of itemspecific arousal at encoding. This result is consistent with the idea that arousal-related modulation during initial processing has downstream consequences on emotional memory retrieval. Supporting this idea, pharmacologically blocking $\beta$-adrenoreceptors only impairs emotional memory recollection when administered prior to encoding but not prior to retrieval (Rimmele et al., 2016). Intriguingly, enhancing NE activity in the rodent amygdala following episodic-like memory training has been shown to pre- 


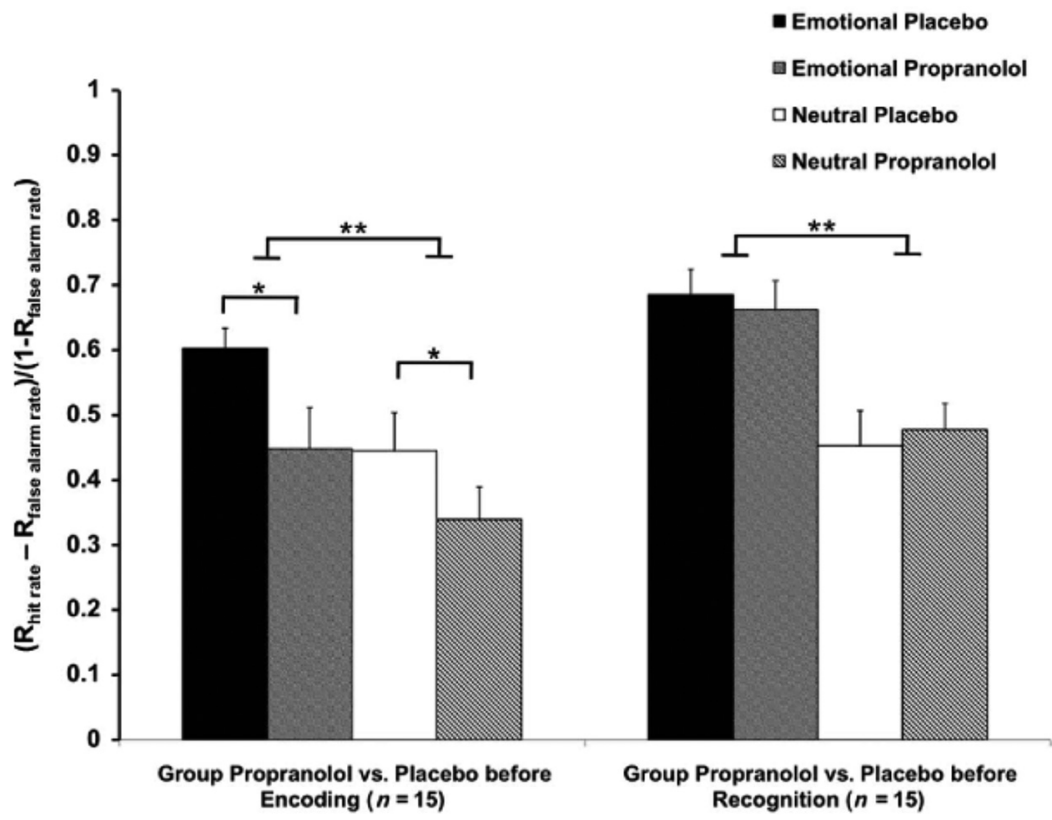

Figure 2. $\beta$-Adrenoreceptor blockade impairs emotional memory recollection at encoding but not at retrieval. Emotion led to enhanced recollection of photographs when memory was probed $24 \mathrm{~h}$ after learning. Administration of $80 \mathrm{mg}$ of propranolol led to decreased recollection for both emotional and neutral stimuli, whereas propranolol had no effect on emotional or neutral recollection (data and figure from Rimmele et al., 2016). Asterisks indicate statistical significance.

serve hippocampal-dependent retrieval and accurate memory 28 d later, suggesting that emotional memories may retain their vividness via NE in the amygdala (Atucha et al., 2017). Thus, without sustaining NE levels after emotional learning and during consolidation, arousal may not exert as strong of an effect on emotional memory.

Together, these findings suggest that the predominant mechanism driving arousal's influence on emotional memory may reflect NE's more general role in cognitive selectivity and attention. Recent theoretical and empirical work suggest that NE release biases both attention and memory to favor high priority (e.g., goal-relevant or perceptually salient) mental representations at the expense of lower priority information irrespective of valence (Lee et al., 2014, 2018; Markovic et al., 2014; Nielsen et al., 2015; Mather et al., 2016; Clewett et al., 2017a,b, 2018a). From this perspective, increased LC-NE activity under arousal promotes the selective encoding of contextual details that are within the focus of attention, binding only the most important aspects of an experience into longterm episodic memory.

These neurocognitive models also suggest that the vivid recollection of emotional events is not determined by negative valence, per se, but rather by synergistic interactions between arousal and any form of stimulus salience at the time NE is released (Mather et al., 2016). Combined rodent and human findings suggest that these strengthened processing-stage interactions may relate more to closely to arousal and LC-NE system activation rather than valence alone. Similar to negative stimuli, activity in the NE-LC system may support sensory recapitulation for positive memoranda. LC responses are not limited to exposure to negative events but also generalize to positive events that are highly arousing or motivating (Berridge and Waterhouse, 2003; Bouret and Sara, 2004; Sara, 2009; Bouret and Richmond, 2015; Todd et al., 2015). Supporting an arousal-based versus valencebased account of emotional memory encoding and retrieval, positive stimuli tend to show neurobehavioral effects similar to negative stimuli in states of putatively greater LC-NE engagement. For example, positive and negative events engage the amygdala to a similar degree under stress, an elevated state of arousal (Cousijn et al., 2010). Broadly, these results align with evidence that amygdala activity also supports the encoding of positive stimuli (Hamann and Mao, 2002). Accordingly, retrieving positive memories should also be associated with enhanced sensory recapitulation processes if those events are arousing enough to activate LC-NE systems at encoding.

Research further suggests that the degree to which positive emotional stimuli (i.e., IAPS images) engage the amygdala is modulated by arousal (Bonnet et al., 2015), and amygdala activation has been linked to emotional memory enhancements for both positive and negative arousing stimuli (Canli et al., 2000; Kensinger and Corkin, 2003). Increased amygdala activity also supports more focused encoding of both positive and negative stimuli, such that fewer peripheral contextual details are later remembered (Mickley and Kensinger, 2008). Stronger coupling between higher arousal ratings and higher emotional memory confidence have also been observed for both positive and negative valences in individuals with a NE gene variant associated with higher NE availability (Todd et al., 2013a). In a separate fMRI study, individuals with this genetic variant exhibited equivalent increases in 
the recall of both positive and negative photographs (de Quervain et al., 2007). While this neurogenetic work suggests a link between NE levels and the subjective quality of memory, this evidence is still indirect. Thus, more direct manipulations of LC-NE system activity (e.g., pharmacology) are needed to validate this relationship.

In sum, these data lend credence to the idea that the degree of arousal-induced NE release and amygdala activity at encoding, regardless of valence, leads to greater memory vividness and recollection during later retrieval. Thus, differences in sensory cortex reinstatement for positive and negative memoranda could emerge in a quantitative fashion (i.e., the same neural pattern but to a lesser or greater extent) given relative differences in arousalrelated engagement of the LC-NE system.

\section{Do mechanisms beyond arousal account for} differences across positive and negative memories?

Above, we described how differences in arousal across positive and negative memoranda may result in differential engagement of the LC-NE system, which leads to differences in subsequent sensory recapitulation and recollection. Yet non-arousal-related factors may also contribute to memory processing, which may explain additional variance in studies reporting valence-dependent differences in sensory cortex reinstatement.

In the following section, we outline a proposal that behavioral activation is an additional factor that shapes neural recapitulation at retrieval. In contrast to purely arousal-driven effects, we argue the behavioral activation may drive the formation of more multiplexed and integrated memory representations. Critically, previous work has only begun to explore the influence of behavioral activation on memory formation. Thus, in the following sections, we describe preliminary and supporting evidence that behavioral activation supports higher-order sensory recapitulation in areas that include more anterior portions of the ventral visual stream and those that integrate multimodal sensory information, such as the parietal cortex, medial PFC, and lateral PFC. We also provide testable hypotheses. Within this expanded framework, differences in reinstatement of sensory cortical activity patterns across positive and negative memoranda could result either from quantitative differences (i.e., how much arousal is elicited) or qualitative differences (i.e., the balance between arousal and/or behavioral activation).

\section{Behavioral activation as a mechanism supporting memory integration}

Here, we operationalize behavioral activation as a motivational state of increased vigor and energization that promotes exploration and novelty-seeking (Alcaro et al., 2007; Düzel et al., 2010; Salamone et al., 2016). These family of related behaviors result in individuals actively exploring their surroundings, which has downstream consequences on the number of features individuals attend to in their environment. We propose that increases in behavioral activation and exploration result in a broadening of cognitive processing, thereby promoting the formation of flexible relationships between multiple features of one event (i.e., associating an item with it surrounding contex- tual element) and the integration of new memory traces with existing memories (i.e., updating schematic information or prior knowledge with new information). Critically, this type of mnemonic integration does not necessarily entail encoding a greater quantity of sensory details from an emotional event. In other words, this is not in direct contrast to arousal's attention-narrowing and memorynarrowing effects towards emotional item-focused sensory features (detailed above). Rather, it specifies that enhanced associations between discrete sensory features promote the formation of higher-order cognitive representations of events.

To put this idea in context, imagine viewing a picture of a campsite with the backdrop of a beautiful mountain (Fig. 3). In an item-based framework, cognitive resources may be focused on encoding specific details about one of the tents in the foreground, such as its color and shape. By contrast, in an integrative memory framework, the same amount of cognitive resources may focus on the relationship between each of the tents and the surrounding forest and mountains. This experience may else invoke memories of prior camping trips. Thus, the overall amount of information may be equivalent across these encoding frameworks, but in the integrative framework, memory representations become transformed into a multi-faceted representation of discrete sensory features.

Critically, states of behavioral activation may be biased towards more positive versus negative affective states. For example, a large body of research has linked increased positive affect to behavioral activation (Custers and Aarts, 2005; Marien et al., 2013). Positive affect, particularly in relatively low-arousal states, has been shown to broaden individual's perception, a process which enhances the global processing of events, attention to multiple features of a scene, and the integration of discrete event features (Gasper and Clore, 2002; Fredrickson, 2004; Gable and Harmon-Jones, 2008). All of these processes are intertwined with our definition of behavioral activation. For example, in an experience sampling study, spontaneous retrieval of positive thoughts was shown to predict participant's motivation to actively engage in goaloriented activities (Rice and Fredrickson, 2017). These types of affective states also elicit a more schematic and elaborative mode of processing and encoding (Fredrickson, 2004), perhaps due to their role in promoting self and social functions (Rasmussen and Bernsten, 2009).

Evidence from autobiographical memory studies also suggests that positive emotional experiences stimuli might in fact contain more peripheral information than negative events, at least subjectively, because memory accuracy cannot be verified in these paradigms (Bernsten, 2002; Talarico et al., 2009). In a similar vein, recent work combining an empirical manipulation with mathematical modeling suggests that positive emotion enhance associative memory for word pairs (Madan et al., 2018). What is important to note is that when experimenters attempt to match arousal levels across positive and negative valences (e.g., based on normative ratings), the arousal level is actually relatively moderate/low. This is because negative stimuli tend to be much more arousing than positive 
Experience

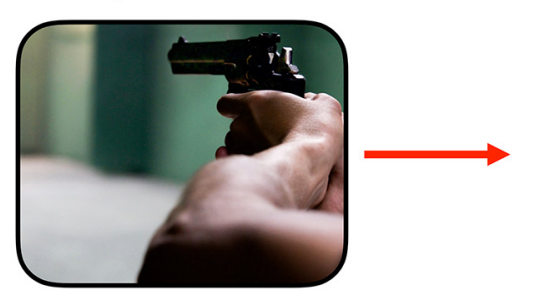

High Physiological Arousal

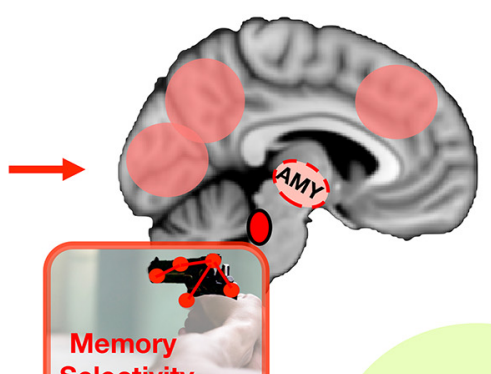

Reinstatement
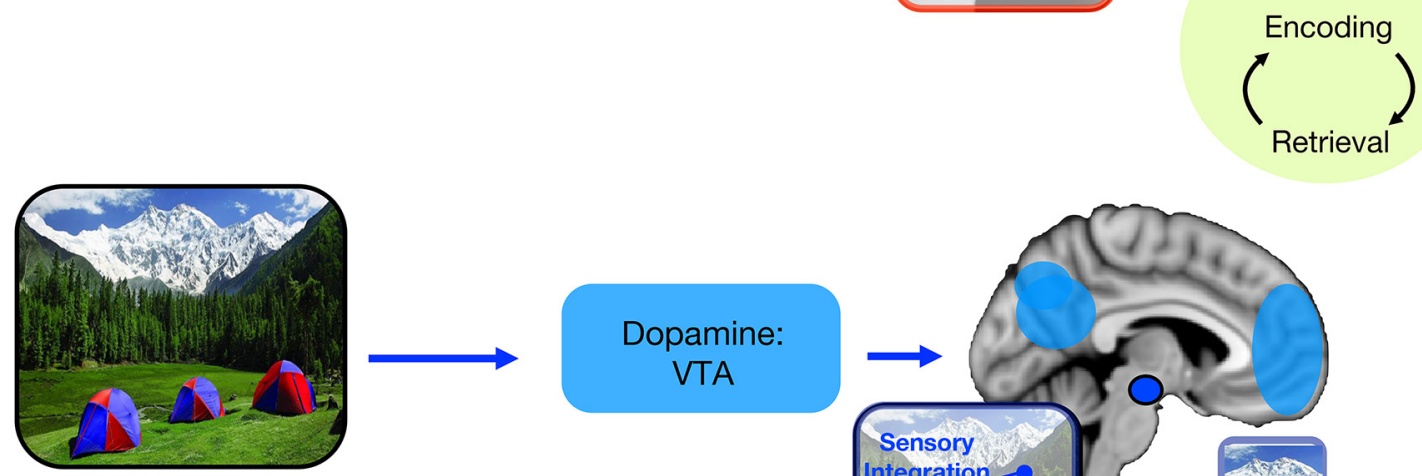

High Behavioral Activation
Norepinephrine: Locus Coeruleus Selectivity

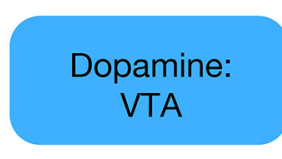

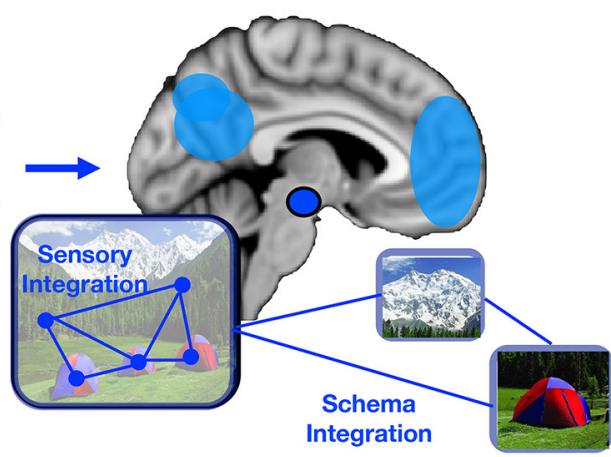

Figure 3. Our proposed neuromodulatory model of emotion-enhanced neural reinstatement and the quality of memory. According to this framework, high arousal levels at encoding activate the LC-NE system (red colors) regardless of valence (top panel). The LC-NE system in turn focuses limited mental resources to process high-priority (e.g., goal-relevant or perceptually salient) and/or emotional item-intrinsic representations in the ventral visual stream, amplifying encoding of a select few details. This narrowing of cognitive and memory processing under arousal is also driven by NE-related activation of the amygdala, which further modulates the selective processing of only the most salient sensory representations. This amygdala-related network may be especially important for enhancing the subjective quality of emotional memory recollection (i.e., memory confidence and vividness). Activation of the LC-NE system during post-encoding periods of consolidation will also amplify the preferential processing of salient event features even further, in part through amygdala modulation (dashed red circle; region is more lateral than depicted). By contrast, behavioral activation, defined as a motivational state of increased vigor, exploration, and novelty seeking, should recruit greater activity in the DA system that is anchored in the VTA (blue colors). In contrast to the LC-NE system, DA modulation should instead broaden the scope of cognitive processing for positive events at encoding (particularly with low arousal), leading to more integrated sensory and mnemonic representations in high-order sensory cortex and PFC. This broadening effect may be amplified by DA activation during offline consolidation and/or neural reactivation, leading to greater integration between positive memories (e.g., associative memory) and existing memory/schema in represented in PFC (blue links to other memories). In sum, engaging these different neuromodulatory systems during early stages of episodic memory should propagate downstream to enhance reinstatement processes in encodingrelated sensory cortex during later retrieval (green circle). As such, which brain system becomes engaged at encoding may be the ultimate determinant of post-encoding patterns of neural reinstatement and the quality of emotional memory recollection.

stimuli. Thus, we would expect that behavioral activation and its resulting cognitive broadening effects would prevail at relatively lower arousal states, whereas higher levels of arousal will elicit cognitive narrowing and greater memory selectivity.

While positive stimuli may be biased towards eliciting behavioral activation, it is critical to note that behavioral activation is not exclusively engaged by positive valence stimuli. Rather behavioral activation may be better aligned to the goal orientation of an individual in response to an emotional item or context. For example, studies have shown that when threats are more distant, individuals goal orientation may be directed towards active avoidance, which relies on behavioral activation and mesolimbic sys- tem (DA) engagement (Fanselow, 1994; Mobbs et al., 2009). However, when threats are more proximal to an individual, goal orientation may be directed towards defensive responses such as startle and freezing, in which circuits associated with behavioral activation are inhibited (Fanselow, 1994; Mobbs et al., 2009). One interpretation of these effects is that more proximal threats elicit greater physiological arousal, whereas lower arousal states allow the cognitive broadening effects of behavioral activation to prevail.

Our approach of characterizing behavioral activation to understand sensory reinstatement and both subjective/ objective aspects of emotional memories parallels several theories of emotion and motivation. For instance, our 
ideas dovetail with the evidence suggesting that goal orientation may modulate the scope of attention and memory processing (Gable and Harmon-Jones, 2011; Harmon-Jones et al., 2013; Kaplan, 2013), with pre-goal attainment states and emotions, such as desire or anger, being associated with cognitive narrowing effects, and post-goal attainment states and emotions, such as happiness or sadness, being associated with cognitive broadening (Harmon-Jones et al., 2013; Kaplan, 2013). Thus, beyond valence, goal orientation may be a key factor for determining the breadth of information that is processed and encoded into long-term memory. Higharousal states have also been likened to the effects of "motivational intensity," or the strength of approach or avoidance emotions, on cognitive narrowing effects (Harmon-Jones et al., 2013). These arousal-like effects on memory are also invariant to emotional valence.

Similarities notwithstanding, the role of goals and emotional states/mood in cognitive processing is somewhat challenging to reconcile with other emotion-cognition studies of declarative memory. Namely, the former class of studies are generally designed to modulate an individual's subjective emotional experience (e.g., sadness, happiness, anger). As such, they might not map clearly onto most of the emotion-cognition memory studies we have reviewed, which tend to query emotional memory for discrete emotional words, sounds, or pictures (for a short discussion, see Harmon-Jones, 2018).

\section{Behavioral activation may drive high-order patterns of neural reinstatement}

The majority of studies characterizing behavioral activation's influence on cognition have focused on early stages of processing, including perception, attention, and encoding. However, these studies lay a strong foundation for making predictions about how behavioral activation may influence memory reinstatement patterns both after encoding and during retrieval. We propose that sensory cortex reinstatement, in the context of behavioral activation, would likely emerge in higher-order brain processing regions, including multi-model sensory cortex and PFC. These neural patterns might therefore reflect high-order cognitive representations that link together multiple elements of sensory events. Although this facet of emotional memory has yet to be tested directly, positive emotional stimuli which tend to be both lower in arousal but also elicit behavioral activation, have been more closely linked to engagement of higher-order sensory cortex, such as more anterior portions of the ventral visual stream (e.g., fusiform gyrus and perirhinal/parahippocampal cortex), as well as PFC regions associated with memory integration and semantic elaboration, such as ventrolateral PFC, orbitofrontal cortex, and medial PFC (Blumenfeld and Ranganath, 2007; Badre, 2008; Preston and Eichenbaum, 2013).

The above framework might help explain why there are some discrepant findings concerning whether patterns of sensory cortex reinstatement are similar across positive and negative valences after encoding. Specifically, these inconsistencies could result from greater variability in the amount of behavioral activation evoked by memoranda. For example, a picture of a smiling face may evoke behavioral activation, activating neurocognitive systems known to facilitate exploration and vigor (Mende-Siedlecki et al., 2013). By contrast, other positive stimuli, such as a picture of a sunset, may induce positive affect in the absence of promoting behavioral activation. Viewed through this lens, emotional stimuli could be varied in their content in a manner that induces qualitatively different types of neural reinstatement, sometimes promoting memory integration while other times promoting selectivity. However, to explore these ideas, future studies need to begin to quantify the extent to which memoranda evoke behavioral activation (see below, Objective measures of arousal, behavioral activation, and neuromodulation).

One way to gain insight into mechanisms by which behavioral activation evokes memory reinstatement is to investigate memory enhancements in response to reward anticipation. Reward anticipation, particularly in the absence of feedback, may serve as a more reliable stimulus to induce behavioral activation than those induced by positive emotional stimuli (e.g., positive IAPS images). Reward anticipation has been shown to increase recollection and source memory during memory retrieval, processes that are thought to rely on enriched retrieval of sensory details from encoding (Wittmann et al., 2005; Adcock et al., 2006; Shigemune et al., 2014). fMRI research in humans has also identified greater postencoding reactivation of sensory stimulus information encoded during reward anticipation (Murty et al., 2017), as well as increased engagement of higher-order sensory and prefrontal cortices by rewarding memoranda during retrieval (Elward et al., 2015). Sensory recapitulation of information encoded during reward anticipation may involve more integrative representations of sensory information (Murty and Adcock, 2017), which would be represented in higher-order sensory and multi-sensory cortex (Schlichting and Preston, 2016).

Notably, these states of reward anticipation detailed above differ from studies which utilize instrumental learning paradigms with performance feedback. In these contexts, individuals may narrow their attention towards the instrumental action necessary to receive positive feedback, thus inhibiting behavioral activation. For example, a recent study characterized how reward influenced memory in the context of a paradigm in which individuals made instrumental actions to receive reward feedback (Gable and Harmon-Jones, 2010). This study found a narrowing of memory and attention during the "pre-goal" phase, i.e., after the reward cue but prior to the instrumental action. Interestingly, this study also found that successfully receiving rewards resulted in "post-goal" emotions which broadened cognitive processing.

\section{The balance between behavioral activation and arousal shapes the scope of memory}

So far, we have detailed how factors associated with increased behavioral activation may elicit a different form of sensory cortex reinstatement than arousal. However, critical questions remain about how these two constructs 


\section{Greater Memory Selectivity}

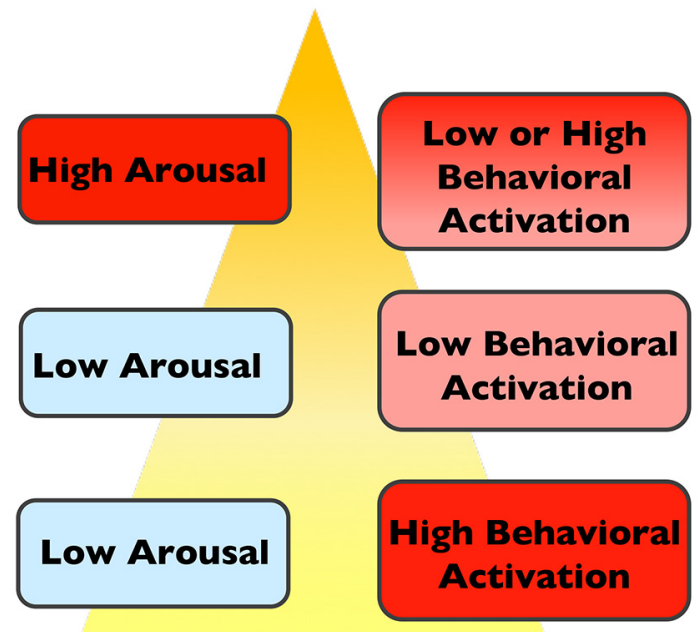

\section{Greater Memory Integration/Broadening}

Figure 4. Putative interactions between arousal and behavioral activation, and their effects on the scope of cognitive and memory processing. According to our framework, there may be interactions between arousal and behavioral activation that influence the balance between memory selectivity versus memory integration. We predict that high-arousal states will elicit greater memory selectivity, regardless of the level of behavioral activation (top row). By contrast, under states of high behavioral activation and low arousal, we predict that there will be greater memory integration and/or broadening (bottom row). These broadening versus narrowing effects occur relative to states of low arousal and low behavioral activation, which we would also characterize as neutral (middle row).

interact. In our view, arousal and behavioral activation are typically orthogonal constructs, but not in all cases. Low levels of arousal but high levels behavioral activation will lead to cognitive and memory broadening. But high arousal will tend to supersede the effects of behavioral activation (or in other words, be invariant to behavioral activation levels), thereby leading to cognitive and memory narrowing. Neutral circumstances, on the other hand, represent states of low behavioral activation and low arousal (Fig. 4).

To illustrate these ideas, consider the following scenarios. Encountering a threatening snake in a zoo may elicit high arousal but not necessarily high behavioral activation, as the threat is confined to a cage; this would theoretically narrow the scope of attention and memory to emotional items, including the snake. By contrast, seeing a threatening snake at a distance on a hike may evoke both high behavioral activation and moderate levels of arousal, such that individuals may explore their environment for escape options; this in turn broadens the scope of attention and memory to process and integrate multiple features and associations in memory. Almost stepping on a snake during a hike, however, may evoke such a level of sympathetic arousal that behavioral activation processes become inhibited. Indeed, this type of behavioral suppression is often observed with threat-induced freezing behavior in both rodents and humans (Fanselow, 1994; Ly et al., 2014).

In line with this example, recent research has shown that the proximity of a threat can modulate defensive responses, which are akin to freezing behavior, suggesting that arousal responses to negative valence stimuli are modulated by other contextual features (Åhs et al., 2015). Similarly, in the domain of positive affect, early exposure to drugs of abuse in rodent models elicit greater behavioral activation, as assayed by exploratory behavior and vigor (Alcaro et al., 2007; Alcaro and Panksepp, 2011); but notably this body of research does not explicitly address arousal. However, in cases where drug cues are extremely salient and induce intense levels of stress and arousal, such as in the context of craving and/or relapse, behaviors may become more habitual and behavioral repertoires become selective only to actions that may elicit acquiring more drugs (Sinha et al., 2003; Sinha, 2013). In line with these interpretations, recent human research has shown that when reward motivation elicits highly arousal states integrative memory can be disrupted, and memory representations can be biased towards cues that directly predict reward at the expense of surrounding contextual details.

From a neuromodulatory point of view, NE levels have also been shown to be elevated during craving and acute withdrawal (Fitzgerald, 2013), and $\beta$-adrenoreceptor activation may facilitate a shift from goal-oriented towards habitual responding under stressful conditions (Schwabe et al., 2011). Together, these studies suggest that high arousal will narrow the scope of attention and memory towards salient information regardless of the level of behavioral activation, perhaps in part by inhibiting exploratory behaviors.

\section{Does neuromodulation explain the putative effects of behavioral activation on neural reinstatement?}

The nature of neural and memory reinstatement patterns evoked by behavioral activation likely involves the engagement of brain systems beyond the LC-NE system. One such candidate mechanism is the mesolimbic DA system. The DA system, which is anchored in the DA substantia nigra and ventral tegmental area (SN/NTA), has been linked to aspects of behavioral activation (Alcaro et al., 2007; Alcaro and Panksepp, 2011). For instance, DA system activation occurs under states of reward motivation that are critical for translating individuals' goals into approach-related actions (Berridge and Robinson, 2003; Wise, 2004). While there is less evidence supporting the mesolimbic DA system's role in memory recollection, we will speculate on this possibility based on evidence that DA promotes associative memory formation.

Accumulated evidence suggests that DA activity contributes to increased sensory processing (Noudoost and Moore, 2011) as well as increased memory reactivation/ recapitulation (Wang and Morris, 2010). Further, the DA system may be driving sensory processing and recapitulation in a different way than the NE system. DA receptors are found throughout the brain, but in very low densities in primary sensory cortical regions; DA axonal projections to 
primary sensory cortex are relatively sparse, especially compared to LC neurons (Jacob and Nienborg, 2018). SN/VTA neurons send dense neuronal projections to higher-order sensory processing regions in anterior portions of the ventral visual stream, which are known to support the relational binding between the elements of experience (Davachi, 2006; Graham et al., 2010). VTA neurons also heavily innervate lateral and medial PFC (Robbins and Arnsten, 2009; Jacob and Nienborg, 2018), which could facilitate more elaborative, self-referential processing and support the integration of incoming information with existing overlapping memories (van Kesteren et al., 2012). Consistent with this possibility, research in rodents and non-human primates show that increased DA signaling can influence prefrontal-mediated mechanisms of attention and downstream engagement of sensory cortex at a higher-order level of processing (Noudoost and Moore, 2011; Jacob and Nienborg, 2018).

This functional neuroanatomy of the DA system makes it well positioned to facilitate the integration of multimodal sensory information under high states of behavioral activation. Research has shown that engagement of the DA system during encoding leads to more integrative memory representations (Shohamy and Wagner, 2008). SN/ VTA activation parallels hippocampal activation during the anticipation of novel stimuli, which has been interpreted as the DA system driving the exploration of new environments (Wittmann et al., 2007). Furthermore, recollection is enhanced for anticipated novel stimuli, suggesting that DA modulation may drive exploratory behaviors that promote encoding of salient, novel events (Wittmann et al., 2007).

There is also fMRI evidence to suggest that DA modulation is important for facilitating associative memory binding during consolidation. Greater post-encoding interactions between the VTA, a central hub of the DA system, and higher-order sensory cortex, such as the perirhinal cortex and category-selective visual cortex, relates to enhanced associative memory (Tompary et al., 2015; Murty et al., 2017, 2018). Although these postencoding fMRI measures do not directly assess brain activity at retrieval, it has been posited that post-encoding reactivation during rest involves similar mechanisms to sensory reinstatement or reactivation at retrieval (Antony et al., 2017).

How memoranda are encoded and consolidated has important consequences for how those experiences are later recapitulated and remembered. In contrast to the attention and memory-narrowing effects of NE under arousal (Mather et al., 2016), emotion-related engagement of the DA system may broaden the scope of cognitive processing during the encoding of rewarding or positive events (Ashby et al., 1999). As described above, however, we would attribute these effects to behavioral activation rather than positive emotion, per se. Insofar as encoding processes dictate which sensory information gets stored and later reactivated at retrieval, this more generalized form of cognitive processing may enhance the associative relationships among different elements of an event (Duncan and Schlichting, 2018). Indeed, at the neural level, activation of the DA system has been shown to enhance associative memory binding and memory generalization both in the context of positive (Wittmann et al., 2005; Murty et al., 2011; Wolosin et al., 2012; Shigemune et al., 2014) and negative events (Clewett et al., 2018b).

Critically, more work is needed to determine the consequences of engaging both arousal and behavioral activation to understand the complex interactions between these neuromodulatory systems during the encoding of motivationally significant events. For example, highly arousing positive stimuli, such as drug cues, could engage both DA and NE systems, given their propensity to evoke both behavioral activation and arousal (Sinha et al., 2003; Koob and Le Moal, 2005; Economidou et al., 2011). Preliminary data suggest that more selective encoding versus broader, higher-order representational encoding will prevail whenever arousal levels are sufficiently elevated (Callan and Schweighofer, 2008; Murty et al., 2011). However, this idea has yet to be tested explicitly, particularly within the context of human emotional memory. An important direction for future research is to explore how these brain systems either work in concert or counter to one another to modulate the quality of emotional memory recollection and corresponding patterns of neural reinstatement in sensory cortex and across the brain.

\section{Testing the factors that drive emotion-related neural reinstatement and memory recollection}

In many ways, the neuromodulatory model we have proposed is preliminary. While grounded in strong theoretical work, more empirical research is needed to test our specific predictions and to compare these predictions against those put forth in the NEVER model. Below we detail some methodological approaches to testing these frameworks.

\section{Controlling differences in arousal and behavioral activation across valences.}

Acquiring participant-specific arousal, valence, as well as behavioral activation assayed through self-report ratings (e.g., BIS/BAS; Carver and White, 1994) as well as behavioral measures (i.e., response vigor) is essential, as there is substantial variability in these ratings across individuals. Some ways to also account for such variability is to integrate item-specific ratings into trial-level linear mixed models, which would allow for direct comparisons of valence versus arousal/behavioral activation effects.

\section{Objective measures of arousal, behavioral activation, and neuromodulation.}

In addition to self-report measures, it will be critical to also assay objective measures of arousal and behavioral activation, as well as their relationship to neuromodulatory system activity. An especially effective way of assessing the effects of arousal on memory is to use more objective physiological measures, including eye-tracking and skin conductance responses. Increasing research in both humans and animals suggests that pupil dilation may be a reliable index of LC activity (Murphy et al., 2014; Varazzani et al., 2015; Joshi et al., 2016; Clewett et al., 2018a). Human research has begun to utilize tools to characterize 
behavioral activation, including characterizing how stimuli invoke vigor, through paradigms using finger tapping (Heerey and Gold, 2007), exploration of visual scenes using eye-tracking (Voss et al., 2017), and the ability of stimuli to evoke approach behaviors using Pavlovian-toInstrumental transfer paradigms (Talmi et al., 2008). Further, research has begun to show that measures of blink rate may index DA activity both at rest and during cognitive tasks (Jongkees and Colzato, 2016). fMRI measures could also be used to measure BOLD signal changes in the LC and VTA/SN during the encoding, consolidation, and retrieval of emotional memories. Finally, up-regulating or down-regulating activity in the NE and DA systems using pharmacological manipulations would also provide evidence of more causal relationships between neuromodulation and emotional memory recapitulation.

\section{Measuring the selectivity of emotional memory recollection.}

One of the key questions we have raised concerns the types of information that are being recapitulated during emotional memory retrieval. To address this issue, future research should carefully manipulate which information is prioritized in attention and memory under emotional circumstances (for discussion, see Mather et al., 2016), and query how memory for high and low priority information relates to sensory recapitulation patterns at retrieval. Similar specificity could be achieved by using more sensitive measures of neural recapitulation with $\mathrm{fMRI}$ data, such as multivoxel pattern similarity analyses. This approach targets the precise correspondence between individual item representations when they are initially encoded and later retrieved (Ritchey et al., 2013). Recent work also shows that similarity in eye movements and fixation patterns across encoding and retrieval may provide an index of item-specific reactivation (Johansson et al., 2012). Interestingly, research also suggests that using picturedrawing to probe the recall of previously studied scene images might also help to reveal the mnemonic content of those experiences (Bainbridge et al., 2019). Using this type of memory probe for emotional scenes could help reveal the types of perceptual information that people encode.

\section{Manipulating study-test retention intervals.}

Research suggests that retention interval may play an important role in modulating recapitulation effects, given that emotion typically has a more pronounced effect on recollection over time (Sharot et al., 2007, 2008; Ritchey et al., 2008; Yonelinas and Ritchey, 2015). Of interest, behavioral work suggests that valence effects on memory may interact with delay and therefore impact the magnitude and/or nature of recapitulation (Pierce and Kensinger, 2011). This facet of recapitulation would be especially relevant, as both the LC-NE and DA systems enhance emotional memory during consolidation (McGaugh, 2004; Shohamy and Adcock, 2010).

\section{Summary of our neuromodulatory model of emotional memory reinstatement}

Our most vivid and enduring memories tend to be emotional. The specific factors and neural processes that drive such detail-laden recollections, however, are somewhat unclear. The recently proposed NEVER model provides an interesting account of this phenomenon by targeting how emotional memory traces laid down at encoding shape the information that is available for later reinstatement. It is posited that, by re-engaging the same perceptual processing mechanisms that operated at encoding, retrieval quite literally reflects the re-experience of prior events. While this model focuses primarily on the role of negative valence in driving this encoding-retrieval overlap, we argue that the bulk of evidence still favors arousal as the key factor driving enhanced emotional memory recollection and vividness. We also provide preliminary evidence to investigate behavioral activation factors as an additional mechanism contributing to the quality of recollection.

At the crux of our model is that arousal and behavioral activation, as well as the neuromodulatory systems they activate, may be better predictors than valence of neural reinstatement patterns and the quality of retrieved emotional memories. From the perspective that encoding and neuromodulatory processes determine the details that are stored in memory, we argue that sensory and high-order cortex reactivation after learning reflects biases in which features of events are reflected in memory (Fig. 3). That is, patterns of reactivation may reflect either a more selective memory representation or something more abstract and generalized.

In our model, an increase in arousal and LC-NE activity promotes the prioritization of the most salient features of an emotional experience at encoding, whereas more mundane sensory information may be initially neglected, and later distorted and/or forgotten. Thus, an inflated sense of confidence and memory vividness for emotional events may be driven by especially strong representations of select details of those events. At present, much research suggests that amygdala may act independently of or in concert with NE activity to amplify these effects across each stage of episodic memory.

In parallel to these encoding mechanisms driven by arousal-related LC-NE system activity, reinstatement effects in higher-order cortex may be driven by behavioral activation and, possibly, activation of the mesolimbic DA system during encoding and post-encoding rest. This form of DA modulation, however, may be qualitatively different from LC-NE system modulation. Under states of elevated DA system engagement, including exploration, novelty, and behavioral vigor, sensory features of the environment may become transformed into more integrated, higher-order representations of events. Positive emotions often co-occur with this class of behavioral responses, which might explain why they also tend to elicit cognitive and memory broadening effects, at least under states of lower arousal or motivational intensity (Harmon-Jones et al., 2013). Further, this integrative mode of processing may promote integration between 
information in the environment and existing memories or schema represented in the PFC.

In sum, the goal of our framework is to move beyond the idea that valence drives differences in reinstatement. Rather, we propose that the degree of arousal and behavioral activation during learning influences downstream patterns of neural reinstatement that manifest qualitative differences in memory (Fig. 4). To reconcile our proposal with valence-related models like NEVER, we propose that negative stimuli may be more likely to evoke higher arousal levels than positive stimuli, and thus could yield greater neural reinstatement in relatively lower-level sensory cortical regions and the amygdala. Conversely, positive stimuli may be more prone to evoke a sense of behavioral activation than negative stimuli, as negative images can often instead promote behavioral inhibition and freezing responses (Azevedo et al., 2005; Blakemore et al., 2016). While speculative, positive events might therefore be more likely to activate the DA system at encoding and consolidation (Murty and Adcock, 2017), leading to better associative memory.

Careful experimental manipulations of the relative priority of multiple features of an experience and an individual's memory for their associations could help unpack the brain and behavioral factors that impact both subjective and objective aspects of emotional memory. To test these predictions, for example, future emotional memory studies would need to systematically vary behavioral activation in addition to arousal across valences, and measure the magnitude and spatial patterns of neural reinstatement following encoding. This could be achieved by modifying traditional emotional memory studies to manipulate exploratory goal states and to quantify the extent to which individual memoranda increase vigor/energization, and/or to manipulate the relative novelty of memoranda. Using multivoxel pattern similarity analyses may be a particularly effective way of quantifying encoding-retrieval overlap for both trial-unique and category-level effects (Ritchey et al., 2013).

\section{Conclusion}

Characterizing the influence of neuromodulators and emotion on recollection has important implications for cognition in healthy individuals and individuals with disorders of emotion. Age-related changes in neuromodulatory systems may have a profound impact on how emotional memories are encoded, stored, and later recollected. These differences may be particularly pronounced in age-related neurodegenerative diseases, such as Alzheimer's and Parkinson's disease, in which decline in the NE and DA systems is significant (Vazey and Aston-Jones, 2012; Robertson, 2013; Mather and Harley, 2016).

The work we have reviewed also has relevance concerning the recapitulation of more naturalistic, real-world memories. In particular, extending this research into the domain of autobiographical memory has clinical relevance to disorders characterized by maladaptive episodic memory binding, such as post-traumatic stress disorder (PTSD). More recently, it has been shown that PTSD is associated with hyper-responsivity of the LC under arousal (Naegeli et al., 2018), suggesting that treatments targeting NE activity could be an effective way of weakening aversive responses and maladaptive memories (Kroes et al., 2016).

Emotions are multifaceted, and there are undoubtedly more complex interactions between arousal, motivation, and cognition, as well as between these neuromodulatory systems, than our preliminary model can address. But we hope that our ideas will invigorate new discussion about the nature of emotional memory and its underlying mechanisms. Through our framework, we encourage cognitive neuroscientists to broaden their focus to include neuromodulation when examining emotion's persistent effects through each stage of episodic memory. Doing so may better inform how bringing different emotional memories to mind impacts how we learn and maintain a sense of wellbeing in the present.

\section{References}

Adcock RA, Thangavel A, Whitfield-Gabrieli S, Knutson B, Gabrieli JDE (2006) Reward-motivated learning: mesolimbic activation precedes memory formation. Neuron 50:507-517.

Adolphs R, Denburg NL, Tranel D (2001) The amygdala's role in long-term declarative memory for gist and detail. Behav Neurosci 115:983-992.

Adolphs R, Tranel D, Buchanan TW (2005) Amygdala damage impairs emotional memory for gist but not details of complex stimuli. Nat Neurosci 8:512-518.

Åns F, Dunsmoor JE, Zielinski D, LaBar KS (2015) Spatial proximity amplifies valence in emotional memory and defensive approachavoidance. Neuropsychologia 70:476-485.

Alcaro A, Panksepp J (2011) The SEEKING mind: primal neuroaffective substrates for appetitive incentive states and their pathological dynamics in addictions and depression. Neurosci Biobehav Rev 35:1805-1820.

Alcaro A, Huber R, Panksepp J (2007) Behavioral functions of the mesolimbic dopaminergic system: an affective neuroethological perspective. Brain Res Rev 56:283-321.

Anderson AK, Yamaguchi Y, Grabski W, Lacka D (2006) Emotional memories are not all created equal: evidence for selective memory enhancement. Learn Mem 13:711-718.

Antony JW, Ferreira CS, Norman KA, Wimber M (2017) Retrieval as a fast route to memory consolidation. Trends Cogn Sci 21:573576.

Ashby FG, Isen AM, Turken AU (1999) A neuropsychological theory of positive affect and its influence on cognition. Psychol Rev 106:529-550.

Atucha E, Vukojevic V, Fornari RV, Ronzoni G, Demougin P, Peter F, Atsak P, Coolen MW, Papassotiropoulos A, McGaugh JL, de Quervain DJ-F, Roozendaal B (2017) Noradrenergic activation of the basolateral amygdala maintains hippocampus-dependent accuracy of remote memory. Proc Natl Acad Sci USA 114:91769181.

Azevedo TM, Volchan E, Imbiriba LA, Rodrigues EC, Oliveira JM, Oliveira LF, Lutterbach LG, Vargas CD (2005) A freezing-like posture to pictures of mutilation. Psychophysiology 42:255-260.

Badre D (2008) Cognitive control, hierarchy, and the rostro-caudal organization of the frontal lobes. Trends Cogn Sci 12:193-200.

Bainbridge WA, Hall EH, Baker Cl (2019) Drawings of real-world scenes during free recall reveal detailed object and spatial information in memory. Nat Commun 10:5.

Bernsten D (2002) Tunnel memories for autobiographical events: central details are remembered more frequently from shocking than from happy experiences. Mem Cognit 30:1010-1020. 
Berridge KC, Robinson TE (2003) Parsing reward. Trends Neurosci 26:507-513.

Berridge CW, Waterhouse BD (2003) The locus coeruleusnoradrenergic system: modulation of behavioral state and statedependent cognitive processes. Brain Res Brain Res Rev 42:3384.

Bisby JA, Burgess N (2013) Negative affect impairs associative memory but not item memory. Learn Mem 21:21-27.

Bisby JA, Burgess N (2017) Differential effects of negative emotion on memory for items and associations, and their relationship to intrusive imagery. Curr Opin Behav Sci 17:124-132.

Blakemore RL, Rieger SW, Vuilleumier P (2016) Negative emotions facilitate isometric force through activation of prefrontal cortex and periaqueductal gray. Neuroimage 124:627-640.

Blumenfeld RS, Ranganath C (2007) Prefrontal cortex and long-term memory encoding: an integrative review of findings from neuropsychology and neuroimaging. Neuroscientist 13:280-291.

Bonnet L, Comte A, Tatu L, Millot J-L, Moulin T, Medeiros de Bustos E (2015) The role of the amygdala in the perception of positive emotions: an "intensity detector." Front Behav Neurosci 9:178.

Bouret S, Sara SJ (2004) Reward expectation, orientation of attention and locus coeruleus-medial frontal cortex interplay during learning. Eur J Neurosci 20:791-802.

Bouret S, Richmond BJ (2015) Sensitivity of locus ceruleus neurons to reward value for goal-directed actions. J Neurosci 35:40054014.

Bowen HJ, Kensinger EA (2017) Recapitulation of emotional source context during memory retrieval. Cortex 91:142-156.

Bowen HJ, Kark SM, Kensinger EA (2018) NEVER forget: negative emotional valence enhances recapitulation. Psychon Bull Rev 25: 860-891. [CrossRef]

Buchanan TW (2007) Retrieval of emotional memories. Psychol Bull 133:761-779.

Callan DE, Schweighofer N (2008) Positive and negative modulation of word learning by reward anticipation. Hum Brain Mapp 29:237249.

Canli T, Zhao Z, Brewer J, Gabrieli JD, Cahill L (2000) Event-related activation in the human amygdala associates with later memory for individual emotional experience. J Neurosci 20:RC99.

Carver CS, White TL (1994) Behavioral inhibition, behavioral activation, and affective responses to impending reward and punishment: the BIS/BAS scales. J Pers Soc Psychol 67:319.

Christianson S-Å, Loftus EF (1991) Remembering emotional events: the fate of detailed information. Cogn Emot 5:81-108.

Clewett D, Sakaki M, Huang R, Nielsen SE, Mather M (2017a) Arousal amplifies biased competition between high and low priority memories more in women than in men: the role of elevated noradrenergic activity. Psychoneuroendocrinology 80:80-91.

Clewett D, Sakaki M, Nielsen S, Petzinger G, Mather M (2017b) Noradrenergic mechanisms of arousal's bidirectional effects on episodic memory. Neurobiol Learn Mem 137:1-14.

Clewett DV, Huang R, Velasco R, Lee T-H, Mather M (2018a) Locus coeruleus activity strengthens prioritized memories under arousal. J Neurosci 38:1558-1574.

Clewett DV, Yi D, Bachman S, Dusmoor J, Phelps EA, Davachi L (2018b) Brain mechanisms by which emotional learning selectively and retroactively enhances memory for related information. International Conference on Learning and Memory, Huntington Beach, CA, April.

Cousijn H, Rijpkema M, Qin S, van Marle HJF, Franke B, Hermans EJ, van Wingen G, Fernández G (2010) Acute stress modulates genotype effects on amygdala processing in humans. Proc Natl Acad Sci USA 107:9867-9872.

Custers R, Aarts H (2005) Positive affect as implicit motivator: on the nonconscious operation of behavioral goals. J Pers Soc Psychol 89:129-142.

Danker JF, Anderson JR (2010) The ghosts of brain states past: remembering reactivates the brain regions engaged during encoding. Psychol Bull 136:87-102.
Danker JF, Tompary A, Davachi L (2017) Trial-by-trial hippocampal encoding activation predicts the fidelity of cortical reinstatement during subsequent retrieval. Cereb Cortex 27:3515-3524.

Davachi $L$ (2006) Item, context and relational episodic encoding in humans. Curr Opin Neurobiol 16:693-700.

de Voogd LD, Fernández G, Hermans EJ (2016) Disentangling the roles of arousal and amygdala activation in emotional declarative memory. Soc Cogn Affect Neurosci 11:1471-1480.

De Quervain DJ-F, Kolassa I-T, Ertl V, Onyut PL, Neuner F, Elbert T, Papassotiropoulos A (2007) A deletion variant of the alpha2badrenoceptor is related to emotional memory in Europeans and Africans. Nat Neurosci 10:1137-1139.

Dew ITZ, Ritchey M, LaBar KS, Cabeza R (2014) Prior perceptual processing enhances the effect of emotional arousal on the neural correlates of memory retrieval. Neurobiol Learn Mem 112:104113.

Dolcos F, LaBar KS, Cabeza R (2004) Interaction between the amygdala and the medial temporal lobe memory system predicts better memory for emotional events. Neuron 42:855-863.

Dougal S, Phelps EA, Davachi L (2007) The role of medial temporal lobe in item recognition and source recollection of emotional stimuli. Cogn Affect Behav Neurosci 7:233-242.

Duncan KD, Schlichting ML (2018) Hippocampal representations as a function of time, subregion, and brain state. Neurobiol Learn Mem 153:40-56.

Düzel E, Bunzeck N, Guitart-Masip M, Düzel S (2010) NOveltyrelated motivation of anticipation and exploration by dopamine (NOMAD): implications for healthy aging. Neurosci Biobehav Rev 34:660-669.

Easterbrook JA (1959) The effect of emotion on cue utilization and the organization of behavior. Psychol Rev 66:183-201.

Eaton T, Anderson AK (2018) Emotional memories and how your life may depend upon them. Behav Brain Sci 41:e11.

Economidou D, Dalley JW, Everitt BJ (2011) Selective norepinephrine reuptake inhibition by atomoxetine prevents cue-induced heroin and cocaine seeking. Biol Psychiatry 69:266-274.

Elward RL, Vilberg KL, Rugg MD (2015) Motivated memories: effects of reward and recollection in the core recollection network and beyond. Cereb Cortex 25:3159-3166.

Fanselow MS (1994) Neural organization of the defensive behavior system responsible for fear. Psychon Bull Rev 1:429-438.

Fitzgerald PJ (2013) Elevated norepinephrine may be a unifying etiological factor in the abuse of a broad range of substances: alcohol, nicotine, marijuana, heroin, cocaine, and caffeine. Subst Abuse 7:171-183.

Fredrickson BL (2004) The broaden-and-build theory of positive emotions. Philos Trans R Soc Lond B Biol Sci 359:1367-1378.

Gable PA, Harmon-Jones E (2008) Approach-motivated positive affect reduces breadth of attention. Psychol Sci 19:476-482.

Gable PA, Harmon-Jones E (2010) The effect of low versus high approach-motivated positive affect on memory for peripherally versus centrally presented information. Emotion 10:599-603.

Gable PA, Harmon-Jones E (2011) Attentional consequences of pregoal and postgoal positive affects. Emotion 11:1358-1367.

Garoff-Eaton RJ, Slotnick SD, Schacter DL (2006) Not all false memories are created equal: the neural basis of false recognition. Cereb Cortex 16:1645-1652.

Gasper K, Clore GL (2002) Attending to the big picture: mood and global versus local processing of visual information. Psychol Sci 13:34-40.

Graham KS, Barense MD, Lee ACH (2010) Going beyond LTM in the MTL: a synthesis of neuropsychological and neuroimaging findings on the role of the medial temporal lobe in memory and perception. Neuropsychologia 48:831-853.

Hamann S, Mao H (2002) Positive and negative emotional verbal stimuli elicit activity in the left amygdala. Neuroreport 13:15-19.

Harmon-Jones E (2018) On motivational influences, moving beyond valence, and integrating dimensional and discrete views of emotion. Cogn Emot 1-8. 
Harmon-Jones E, Gable PA, Price TF (2013) Does Negative Affect Always Narrow and Positive Affect Always Broaden the Mind? Considering the Influence of Motivational Intensity on Cognitive Scope. Curr Dir Psychol Sci 22:301-307.

Heerey EA, Gold JM (2007) Patients with schizophrenia demonstrate dissociation between affective experience and motivated behavior. J Abnorm Psychol 116:268-278.

Hermans EJ, Kanen JW, Tambini A, Fernández G, Davachi L, Phelps EA (2017) Persistence of Amygdala-Hippocampal Connectivity and Multi-Voxel Correlation Structures During Awake Rest After Fear Learning Predicts Long-Term Expression of Fear. Cereb Cortex 27:3028-3041.

Jacob SN, Nienborg H (2018) Monoaminergic neuromodulation of sensory processing. Front Neural Circuits 12:51.

Johansson R, Holsanova J, Dewhurst R, Holmqvist K (2012) Eye movements during scene recollection have a functional role, but they are not reinstatements of those produced during encoding. $J$ Exp Psychol Hum Percept Perform 38:1289-1314.

Jongkees BJ, Colzato LS (2016) Spontaneous eye blink rate as predictor of dopamine-related cognitive function-a review. Neurosci Biobehav Rev 71:58-82.

Joshi S, Li Y, Kalwani RM, Gold JI (2016) Relationships between pupil diameter and neuronal activity in the locus coeruleus, colliculi, and cingulate cortex. Neuron 89:221-234.

Kark SM, Kensinger EA (2015) Effect of emotional valence on retrieval-related recapitulation of encoding activity in the ventral visual stream. Neuropsychologia 78:221-230.

Kaplan RL (2013) Effects of discrete positive emotions on memory: contrasting arousal and goal relevance models. PhD Dissertation, University of California-Irvine.

Kensinger EA (2007) Negative emotion enhances memory accuracy: behavioral and neuroimaging evidence. Curr Dir Psychol Sci 16: 213-218.

Kensinger EA (2009) What factors need to be considered to understand emotional memories? Emot Rev 1:120-121.

Kensinger EA, Corkin S (2003) Memory enhancement for emotional words: are emotional words more vividly remembered than neutral words? Mem Cognit 31:1169-1180.

Kensinger EA, Garoff-Eaton RJ, Schacter DL (2007a) Effects of emotion on memory specificity in young and older adults. J Gerontol B Psychol Sci Soc Sci 62:P208-P215.

Kensinger EA, Garoff-Eaton RJ, Schacter DL (2007b) How negative emotion enhances the visual specificity of a memory. J Cogn Neurosci 19:1872-1887.

Koob GF, Le Moal M (2005) Plasticity of reward neurocircuitry and the "dark side" of drug addiction. Nat Neurosci 8:1442-1444.

Kroes MCW, Schiller D, LeDoux JE, Phelps EA (2016) Translational approaches targeting reconsolidation. Curr Top Behav Neurosci 28:197-230.

LaBar KS, Cabeza R (2006) Cognitive neuroscience of emotional memory. Nat Rev Neurosci 7:54-64.

Lang P, Bradley MM (2007) The international affective picture system (IAPS) in the study of emotion and attention. Handbook of Emotion elicitation and assessment, p 29. New York, NY: Oxford University Press.

Lee T, Greening S, Ueno T, Clewett D, Ponzio A, Sakaki M, Mather M (2018) Arousal increases neural gain via the locus coeruleusnoradrenaline system in younger adults but not in older adults Nat Hum Behav 2:356-366.

Lee TH, Sakaki M, Cheng R, Velasco R, Mather M (2014) Emotional arousal amplifies the effects of biased competition in the brain. Soc Cogn Affect Neurosci 9:2067-2077.

Ly V, Huys QJM, Stins JF, Roelofs K, Cools R (2014) Individual differences in bodily freezing predict emotional biases in decision making. Front Behav Neurosci 8:237. [CrossRef]

Mack ML, Preston AR (2016) Decisions about the past are guided by reinstatement of specific memories in the hippocampus and perirhinal cortex. Neuroimage 127:144-157.
Madan CR, Fujiwara E, Caplan JB, Sommer T (2017) Emotional arousal impairs association-memory: roles of amygdala and hippocampus. Neuroimage 156:14-28.

Madan CR, Scott SME, Kensinger EA (2018) Positive emotion enhances association-memory. Emotion. Advance online publication. Retrieved August 20, 2018.

Maratos EJ, Dolan RJ, Morris JS, Henson RN, Rugg MD (2001) Neural activity associated with episodic memory for emotional context. Neuropsychologia 39:910-920.

Marien H, Aarts H, Custers R (2013) Adaptive control of human action: the role of outcome representations and reward signals. Front Psychol 4:602.

Markovic J, Anderson AK, Todd RM (2014) Tuning to the significant: neural and genetic processes underlying affective enhancement of visual perception and memory. Behav Brain Res 259:229-241.

Mather M (2007) Emotional arousal and memory binding: an objectbased framework. Perspect Psychol Sci 2:33-52.

Mather M, Nesmith K (2008) Arousal-Enhanced Location Memory for Pictures. J Mem Lang 58:449-464.

Mather M, Sutherland MR (2011) Arousal-biased competition in perception and memory. Perspect Psychol Sci 6:114-133.

Mather M, Harley CW (2016) The locus coeruleus: essential for maintaining cognitive function and the aging brain. Trends Cogn Sci 20:214-226.

Mather M, Clewett D, Sakaki M, Harley CW (2016) Norepinephrine ignites local hotspots of neuronal excitation: how arousal amplifies selectivity in perception and memory. Behav Brain Sci 39:e200.

McClelland JL, McNaughton BL, O'Reilly RC (1995) Why there are complementary learning systems in the hippocampus and neocortex: insights from the successes and failures of connectionist models of learning and memory. Psychol Rev 102:419-457.

McGaugh JL (2000) Memory-a centrury of consolidation. Science 287(5451):248-251.

McGaugh JL (2004) The amygdala modulates the consolidation of memories of emotionally arousing experiences. Annu Rev Neurosci 27:1-28.

McGaugh JL (2015) Consolidating memories. Annu Rev Psychol 66:1-24.

McGaugh JL, Roozendaal B (2002) Role of adrenal stress hormones in forming lasting memories in the brain. Curr Opin Neurobiol 12:205-210.

McIntyre CK, McGaugh JL, Williams CL (2012) Interacting brain systems modulate memory consolidation. Neurosci Biobehav Rev 36:1750-1762.

Mende-Siedlecki P, Said CP, Todorov A (2013) The social evaluation of faces: a meta-analysis of functional neuroimaging studies. Soc Cogn Affect Neurosci 8:285-299.

Mickley KR, Kensinger EA (2008) Emotional valence influences the neural correlates associated with remembering and knowing. Cogn Affect Behav Neurosci 8:143-152.

Mickley Steinmetz KR, Addis DR, Kensinger EA (2010) The effect of arousal on the emotional memory network depends on valence. Neuroimage 53:318-324.

Mobbs D, Marchant JL, Hassabis D, Seymour B, Tan G, Gray M, Petrovic P, Dolan RJ, Frith CD (2009) From threat to fear: the neural organization of defensive fear systems in humans. J Neurosci 29:12236-12243.

Murphy PR, O'Connell RG, O'Sullivan M, Robertson IH, Balsters JH (2014) Pupil diameter covaries with BOLD activity in human locus coeruleus. Hum Brain Mapp 35:4140-4154.

Murray BD, Kensinger EA (2013) A review of the neural and behavioral consequences for unitizing emotional and neutral information. Front Behav Neurosci 7:42.

Murty VP, Adcock RA (2017) Distinct medial temporal lobe network states as neural contexts for motivated memory formation. In: The hippocampus from cells to systems, pp 467-501. Cham, Switzerland: Springer

Murty VP, Ritchey M, Adcock RA, LaBar KS (2010) fMRI studies of successful emotional memory encoding: a quantitative metaanalysis. Neuropsychologia 48:3459-3469. 
Murty VP, LaBar KS, Hamilton DA, Adcock RA (2011) Is all motivation good for learning? Dissociable influences of approach and avoidance motivation in declarative memory. Learn Mem 18:712-717.

Murty VP, Tompary A, Adcock RA, Davachi L (2017) Selectivity in postencoding connectivity with high-level visual cortex is associated with reward-motivated memory. J Neurosci 37:537-545.

Murty VP, DuBrow S, Davachi L (2018) Decision-making increases episodic memory via postencoding consolidation. J Cogn Neurosci 1-10.

Naegeli C, Zeffiro T, Piccirelli M, Jaillard A, Weilenmann A, Hassanpour K, Schick M, Rufer M, Orr SP, Mueller-Pfeiffer C (2018) Locus coeruleus activity mediates hyperresponsiveness in posttraumatic stress disorder. Biol Psychiatry 83:254-262.

Nielsen SE, Barber SJ, Chai A, Clewett DV, Mather M (2015) Sympathetic arousal increases a negative memory bias in young women with low sex hormone levels. Psychoneuroendocrinology 62:96-106.

Noudoost B, Moore T (2011) The role of neuromodulators in selective attention. Trends Cogn Sci 15:585-591.

Nyberg L, Habib R, Mclntosh AR, Tulving E (2000) Reactivation of encoding-related brain activity during memory retrieval. Proc Natl Acad Sci USA 97:11120-11124.

Ochsner KN (2000) Are affective events richly recollected or simply familiar? The experience and process of recognizing feelings past. J Exp Psychol Gen 129:242-261.

Phelps EA, Sharot T (2008) How (and why) emotion enhances the subjective sense of recollection. Curr Dir Psychol Sci 17:147-152.

Pierce BH, Kensinger EA (2011) Effects of emotion on associative recognition: valence and retention interval matter. Emotion 11: 139-144.

Preston AR, Eichenbaum H (2013) Interplay of hippocampus and prefrontal cortex in memory. Curr Biol 23:R764-R773.

Rasmussen AS, Berntsen D (2009) Emotional valence and the functions of autobiographical memories: positive and negative memories serve different functions. Mem Cognit 37:477-492.

Rice EL, Fredrickson BL (2017) Do positive spontaneous thoughts function as incentive salience? Emotion 17:840-855.

Rimmele U, Davachi L, Petrov R, Dougal S, Phelps EA (2011) Emotion enhances the subjective feeling of remembering, despite lower accuracy for contextual details. Emotion 11:553-562.

Rimmele U, Davachi L, Phelps EA (2012) Memory for time and place contributes to enhanced confidence in memories for emotional events. Emotion 12:834-846.

Rimmele U, Lackovic SF, Tobe RH, Leventhal BL, Phelps EA (2016) Beta-adrenergic blockade at memory encoding, but not retrieval, decreases the subjective sense of recollection. J Cogn Neurosci 28:895-907.

Ritchey M, Dolcos F, Cabeza R (2008) Role of amygdala connectivity in the persistence of emotional memories over time: an eventrelated FMRI investigation. Cereb Cortex 18:2494-2504.

Ritchey M, Wing EA, LaBar KS, Cabeza R (2013) Neural similarity between encoding and retrieval is related to memory via hippocampal interactions. Cereb Cortex 23:2818-2828.

Robbins TW, Arnsten AFT (2009) The neuropsychopharmacology of fronto-executive function: monoaminergic modulation. Annu Rev Neurosci 32:267-287.

Robertson IH (2013) A noradrenergic theory of cognitive reserve: implications for Alzheimer's disease. Neurobiol Aging 34:298-308.

Roozendaal B, Bohus B, McGaugh JL (1996) Dose-dependent suppression of adrenocortical activity with metyrapone: effects on emotion and memory. Psychoneuroendocrinology 21:681-693.

Roozendaal B, Castello NA, Vedana G, Barsegyan A, McGaugh JL (2008) Noradrenergic activation of the basolateral amygdala modulates consolidation of object recognition memory. Neurobiol Learn Mem 90:576-579.

Sakaki M, Fryer K, Mather M (2014) Emotion strengthens high priority memory traces but weakens low priority memory traces. Psychol Sci 25:387-395.
Salamone JD, Pardo M, Yohn SE, López-Cruz L, SanMiguel N, Correa M (2016) Mesolimbic dopamine and the regulation of motivated behavior. Curr Top Behav Neurosci 27:231-257.

Sara SJ (2009) The locus coeruleus and noradrenergic modulation of cognition. Nat Rev Neurosci 10:211-223.

Schlichting ML, Preston AR (2016) Hippocampal-medial prefrontal circuit supports memory updating during learning and postencoding rest. Neurobiol Learn Mem 134:91-106.

Schwabe L, Höffken O, Tegenthoff M, Wolf OT (2011) Preventing the stress-induced shift from goal-directed to habit action with a $\beta$-adrenergic antagonist. J Neurosci 31:17317-17325.

Segal SK, Cahill L (2009) Endogenous noradrenergic activation and memory for emotional material in men and women. Psychoneuroendocrinology 34:1263-1271.

Sharot T, Delgado MR, Phelps EA (2004) How emotion enhances the feeling of remembering. Nat Neurosci 7:1376-1380.

Sharot T, Verfaellie M, Yonelinas AP (2007) How emotion strengthens the recollective experience: a time-dependent hippocampal process. PLoS One 2:e1068.

Sharot T, Davidson ML, Carson MM, Phelps EA (2008) Eye movements predict recollective experience. PLoS One 3:e2884.

Shigemune Y, Tsukiura T, Kambara T, Kawashima R (2014) Remembering with gains and losses: effects of monetary reward and punishment on successful encoding activation of source memories. Cereb Cortex 24:1319-1331.

Shohamy D, Wagner AD (2008) Integrating memories in the human brain: hippocampal-midbrain encoding of overlapping events. Neuron 60:378-389.

Shohamy D, Adcock RA (2010) Dopamine and adaptive memory. Trends Cogn Sci 14:464-472.

Sinha R (2013) The clinical neurobiology of drug craving. Curr Opin Neurobiol 23:649-654.

Sinha R, Talih M, Malison R, Cooney N, Anderson GM, Kreek MJ (2003) Hypothalamic-pituitary-adrenal axis and sympatho-adrenomedullary responses during stress-induced and drug cue-induced cocaine craving states. Psychopharmacology (Berl) 170:62-72.

Smith APR, Stephan KE, Rugg MD, Dolan RJ (2006) Task and content modulate amygdala-hippocampal connectivity in emotional retrieval. Neuron 49:631-638.

Southwick SM, Davis M, Horner B, Cahill L, Morgan CA, Gold PE, Bremner JD, Charney DC (2002) Relationship of enhanced norepinephrine activity during memory consolidation to enhanced longterm memory in humans. Am J Psychiatry 159:1420-1422.

Steinmetz KRM, Kensinger EA (2013) The emotion-induced memory trade-off: more than an effect of overt attention? Mem Cognit 41:69-81.

Sterpenich V, D'Argembeau A, Desseilles M, Balteau E, Albouy G, Vandewalle G, Degueldre C, Luxen A, Collette F, Maquet P (2006) The locus ceruleus is involved in the successful retrieval of emotional memories in humans. J Neurosci 26:7416-7423.

Strange BA, Dolan RJ (2004) Beta-adrenergic modulation of emotional memory-evoked human amygdala and hippocampal responses. Proc Natl Acad Sci USA 101:11454-11458.

Takashima A, van der Ven F, Kroes MCW, Fernández G (2016) Retrieved emotional context influences hippocampal involvement during recognition of neutral memories. Neuroimage 143:280-292.

Talarico JM, Berntsen D, Rubin DC (2009) Positive emotions enhance recall of peripheral details. Cogn Emot 23:380-398.

Talmi D, Seymour B, Dayan P, Dolan RJ (2008) Human pavlovianinstrumental transfer. J Neurosci 28:360-368.

Todd RM, Talmi D, Schmitz TW, Susskind J, Anderson AK (2012) Psychophysical and neural evidence for emotion-enhanced perceptual vividness. J Neurosci 32:11201-11212.

Todd RM, Müller DJ, Lee DH, Robertson A, Eaton T, Freeman N, Palombo DJ, Levine B, Anderson AK (2013a) Genes for emotionenhanced remembering are linked to enhanced perceiving. Psychol Sci 24:2244-2253.

Todd RM, Schmitz TW, Susskind J, Anderson AK (2013b) Shared neural substrates of emotionally enhanced perceptual and mnemonic vividness. Front Behav Neurosci 7:40. 
Todd RM, Ehlers MR, Müller DJ, Robertson A, Palombo DJ, Freeman N, Levine B, Anderson AK (2015) Neurogenetic variations in norepinephrine availability enhance perceptual vividness. J Neurosci 35:6506-6516.

Tompary A, Duncan K, Davachi L (2015) Consolidation of associative and item memory is related to post-encoding functional connectivity between the ventral tegmental area and different medial temporal lobe subregions during an unrelated task. J Neurosci 35:7326-7331.

Tompary A, Duncan K, Davachi L (2016) High-resolution investigation of memory-specific reinstatement in the hippocampus and perirhinal cortex. Hippocampus 26:995-1007.

Touryan SR, Marian DE, Shimamura AP (2007) Effect of negative emotional pictures on associative memory for peripheral information. Memory 15:154-166.

Tully K, Bolshakov VY (2010) Emotional enhancement of memory: how norepinephrine enables synaptic plasticity. Mol Brain 3:15.

Tulving E, Markowitsch HJ (1998) Episodic and declarative memory: role of the hippocampus. Hippocampus 8:198-204.

Van Kesteren MTR, Ruiter DJ, Fernández G, Henson RN (2012) How schema and novelty augment memory formation. Trends Neurosci 35:211-219.

Varazzani C, San-Galli A, Gilardeau S, Bouret S (2015) Noradrenaline and dopamine neurons in the reward/effort trade-off: a direct electrophysiological comparison in behaving monkeys. J Neurosci 35:7866-7877.

Vazey EM, Aston-Jones G (2012) The emerging role of norepinephrine in cognitive dysfunctions of Parkinson's disease. Front Behav Neurosci 6:48.
Ventura-Bort C, Löw A, Wendt J, Moltó J, Poy R, Dolcos F, Hamm AO, Weymar M (2016) Binding neutral information to emotional contexts: brain dynamics of long-term recognition memory. Cogn Affect Behav Neurosci 16:234-247.

Voss JL, Bridge DJ, Cohen NJ, Walker JA (2017) A closer look at the hippocampus and memory. Trends Cogn Sci 21:577-588

Wang SH, Morris RGM (2010) Hippocampal-neocortical interactions in memory formation, consolidation, and reconsolidation. Annu Rev Psychol 61:49-79.

Wheeler ME, Petersen SE, Buckner RL (2000) Memory's echo: vivid remembering reactivates sensory-specific cortex. Proc Natl Acad Sci USA 97:11125-11129.

Wise RA (2004) Dopamine, learning and motivation. Nat Rev Neurosci 5:483-494.

Wittmann BC, Schott BH, Guderian S, Frey JU, Heinze H-J, Düzel E (2005) Reward-related FMRI activation of dopaminergic midbrain is associated with enhanced hippocampus-dependent long-term memory formation. Neuron 45:459-467.

Wittmann BC, Bunzeck N, Dolan RJ, Düzel E (2007) Anticipation of novelty recruits reward system and hippocampus while promoting recollection. Neuroimage 38:194-202.

Wolosin SM, Zeithamova D, Preston AR (2012) Reward modulation of hippocampal subfield activation during successful associative encoding and retrieval. J Cogn Neurosci 24:1532-1547.

Xie W, Zhang W (2017) Negative emotion enhances mnemonic precision and subjective feelings of remembering in visual long-term memory. Cognition 166:73-83.

Yonelinas AP, Ritchey M (2015) The slow forgetting of emotional episodic memories: an emotional binding account. Trends Cogn Sci 19:259-267. 